\title{
BROWN MEASURES OF UNBOUNDED OPERATORS AFFILIATED WITH A FINITE VON NEUMANN ALGEBRA
}

\author{
UFFE HAAGERUP and HANNE SCHULTZ*
}

(Dedicated to the memory of Gert K. Pedersen)

\begin{abstract}
In this paper we generalize Brown's spectral distribution measure to a large class of unbounded operators affiliated with a finite von Neumann algebra. Moreover, we compute the Brown measure of all unbounded $R$-diagonal operators in this class. As a particular case, we determine the Brown measure $z=x y^{-1}$, where $(x, y)$ is a circular system in the sense of Voiculescu, and we prove that for all $n \in \mathrm{N}, z^{n} \in L^{p}(\mathcal{M}, \tau)$ if and only if $0<p<\frac{2}{n+1}$.
\end{abstract}

\section{Introduction}

Let $\mathcal{M}$ be a finite von Neumann algebra with a faithful, normal, tracial state $\tau$, and let

$$
\Delta(T)=\exp \left(\int_{0}^{\infty} \log t d \mu_{|T|}(t)\right)
$$

denote the corresponding Fuglede-Kadison determinant. L. G. Brown proved in [3] that for every $T \in \mathcal{M}$, there exists a unique, compactly supported measure $\mu_{T} \in \operatorname{Prob}(\mathrm{C})$ with the property that

$$
\log \Delta(T-\lambda \mathbf{1})=\int_{\mathrm{C}} \log |z-\lambda| d \mu_{T}(z), \quad \lambda \in \mathrm{C} .
$$

This measure is called Brown's spectral distribution measure (or just the Brown measure) of $T$. It was computed in a number of special cases in [9], [2], [5], and [1]. In particular, it was proven in [9, Theorem 4.5] that if $T \in \mathcal{M}$ is $R$-diagonal in the sense of Nica and Speicher [16], then $\mu_{T}$ can be determined from the $S$-transform of the distribution $\mu_{|T|^{2}}$. For simplicity, assume that $T \in \mathcal{M}$ is an $R$-diagonal element which is not proportional to a unitary and for which

\footnotetext{
* Supported by the Danish National Research Foundation.

Received May 14, 2006.
} 
$\operatorname{ker}(T)=0$. Then $\mu_{T}$ is the unique probability measure on $\mathrm{C}$ which is invariant under the rotations $z \mapsto \gamma z, \gamma \in \mathrm{T}$, and which satisfies

$$
\mu_{T}\left(B\left(0, \mathscr{S}_{\mu_{|T|^{2}}}(t-1)^{-\frac{1}{2}}\right)\right)=t, \quad 0<t<1 .
$$

In this paper we extend the Brown measure to all operators in the set $\mathcal{M}^{\Delta}$ of closed, densely defined operators $T$ affiliated with $\mathscr{M}$ satisfying

$$
\int_{0}^{\infty} \log ^{+} t d \mu_{|T|}(t)<\infty
$$

where $\log ^{+} t=\max \{\log t, 0\}$. Moreover, we extend [9, Theorem 4.5] to all $R$-diagonal operators in $\mathscr{M}^{\Delta}$. Finally, we will study a particular example of an unbounded $R$-diagonal element, namely the operator $z=x y^{-1}$, where $(x, y)$ is a circular system in the sense of Voiculescu.

The material in this paper is organized as follows: In section 2 we introduce the class $\mathscr{M}^{\Delta}$ and generalize the Brown measure to all $T \in \mathscr{M}^{\Delta}$ by proving, that for such $T$, there is a unique $\mu_{T} \in \operatorname{Prob}(\mathrm{C})$ satisfying

$$
\int_{C} \log ^{+}|z| d \mu_{T}(z)<\infty
$$

and

$$
\log \Delta(T-\lambda \mathbf{1})=\int_{\mathrm{C}} \log |z-\lambda| d \mu_{T}(z), \quad \lambda \in \mathrm{C} .
$$

Moreover, we extend Weil's inequality

$$
\int_{C}|z|^{p} d \mu_{T}(z) \leq\|T\|_{p}^{p}
$$

to all $T \in L^{p}(\mathscr{M}, \tau)$. The main results in section 2 are stated in the appendix of Brown's paper [3] without proofs or with very sketchy proofs. Since the results of the remaining sections of this paper and of our forthcoming paper [10] rely heavily on these statements, we have decided to include complete proofs. We will follow a different route than the one outlined in [3]. For instance, we do not use the functions $\Lambda_{t}(T)$ and $s_{T}(t)$ from [3, section 1].

In section 3 we introduce unbounded $R$-diagonal operators and we prove the following generalization of [9, section 3]: The powers $\left(S^{n}\right)_{n=1}^{\infty}$ of an $R$ diagonal operator are $R$-diagonal, and the sum $S+T$ and the product $S T$ of $*$-free $R$-diagonal operators are again $R$-diagonal. Moreover,

$$
\begin{aligned}
\mu_{\left|S^{n}\right|^{2}} & =\mu_{|S|^{2}}^{\bigotimes n}, \\
\tilde{\mu}_{|S+T|} & =\tilde{\mu}_{|S|} \boxplus \tilde{\mu}_{|T|}, \\
\mu_{|S T|^{2}} & =\mu_{|S|^{2}} \otimes \mu_{|T|^{2}},
\end{aligned}
$$


where $\tilde{\mu}=\frac{1}{2}(\mu+\check{\mu})$ denotes the symmetrization of a measure $\mu \in \operatorname{Prob}(\mathbf{R})$, and $\boxplus$ ( $\otimes$, resp.) denotes the additive (multiplicative, resp.) free convolution of measures (cf. [4]). These results are applied in section 4 to determine the Brown measure of $R$-diagonal operators in $\mathcal{M}^{\Delta}$.

In section 5 we consider the operator $z=x y^{-1}$, where $(x, y)$ is a circular system in the sense of Voiculescu, and we prove that the Brown measure of $z$ is given by

$$
d \mu_{z}(s)=\frac{1}{\pi\left(1+|s|^{2}\right)^{2}} d \operatorname{Re} s d \operatorname{Im} s .
$$

Moreover, we show that for all $n \in \mathrm{N}, z^{n}, z^{-n} \in L^{p}(\mathcal{M}, \tau)$ iff $0<p<\frac{2}{n+1}$, and when this holds,

$$
\left\|z^{n}\right\|_{p}^{p}=\left\|z^{-n}\right\|_{p}^{p}=\frac{(n+1) \sin \left(\frac{\pi p}{2}\right)}{\sin \left(\frac{(n+1) \pi p}{2}\right)},
$$

and

$$
\left\|\left(z^{n}-\lambda \mathbf{1}\right)^{-1}\right\|_{p} \leq\left\|z^{-n}\right\|_{p}, \quad \lambda \in \mathrm{C} .
$$

The last two formulas play a key role in our forthcoming paper [10] on invariant subspaces for operators in a general $\mathrm{II}_{1}$-factor.

\section{The Brown measure of certain unbounded operators}

In [3, Appendix] Brown described in outline how to define a Brown measure for certain unbounded operators affiliated with $\mathscr{M}$, where $\mathscr{M}$ is a von Neumann algebra equipped with a faithful, normal, semifinite trace.

In this section we give a more detailed exposition on the subject in the case where $\mathscr{M}$ is a finite von Neumann algebra with faithful, tracial state $\tau$. To be more explicit, we show how one can extend the definition of the Brown measure to a class $\mathscr{M}^{\Delta}$ of closed, densely defined operators affiliated with $\mathscr{M}$. We also prove that many of the properties of the Brown measure for bounded operators carry over to the unbounded case.

We let $\tilde{\mathscr{M}}$ denote the set of closed, densely defined operators affiliated with $\mathcal{M}$. Recall that every operator $T \in \tilde{\mathscr{M}}$ has a polar decomposition

$$
T=U|T|=U \int_{0}^{\infty} t d E_{|T|}(t),
$$

where $U \in \mathscr{M}$ is a unitary, and the spectral measure $E_{|T|}$ takes values in $\mathscr{M}$. In particular, for $T \in \tilde{\mathscr{M}}$ we may define $\mu_{|T|} \in \operatorname{Prob}(\mathrm{R})$ by

$$
\mu_{|T|}(B)=\tau\left(E_{|T|}(B)\right), \quad(B \in \mathrm{B}) .
$$


Definition 2.1. We denote by $\mathcal{M}^{\Delta}$ the set of operators $T \in \tilde{\mathscr{M}}$ fulfilling the condition

$$
\tau\left(\log ^{+}|T|\right)=\int_{0}^{\infty} \log ^{+}(t) d \mu_{|T|}(t)<\infty .
$$

For $T \in \mathscr{M}^{\Delta}$, the integral

$$
\int_{0}^{\infty} \log t d \mu_{|T|}(t) \in \mathrm{R} \cup\{-\infty\}
$$

is well-defined, and we define the Fuglede-Kadison determinant of $T, \Delta(T) \in$ $[0, \infty)$, by

$$
\Delta(T)=\exp \left(\int_{0}^{\infty} \log t d \mu_{|T|}(t)\right)
$$

Note that for $T \in \mathcal{M}, \Delta(T)$ is the usual Fuglede-Kadison determinant of $T$.

Remark 2.2. If $T \in L^{p}(\mathcal{M}, \tau)$ for some $p \in(0, \infty)$, then

$$
\int_{0}^{\infty} t^{p} d \mu_{|T|}(t)<\infty
$$

implying that

$$
\int_{1}^{\infty} \log t d \mu_{|T|}(t)=\frac{1}{p} \int_{1}^{\infty} \log \left(t^{p}\right) d \mu_{|T|}(t) \leq \frac{1}{p} \int_{1}^{\infty} t^{p} d \mu_{|T|}(t)<\infty,
$$

and hence $T \in \mathscr{M}^{\Delta}$.

Lemma 2.3. If $T \in \mathscr{M}^{\Delta}$ and $\Delta(T)>0$, then $T$ is invertible in $\tilde{\mathscr{M}}, T^{-1} \in$ $\mathcal{M}^{\Delta}$, and $\Delta\left(T^{-1}\right)=\frac{1}{\Delta(T)}$.

Proof. If $T \in \mathcal{M}^{\Delta}$ and $\Delta(T)>0$, then

$$
\int_{0}^{1}|\log t| d \mu_{|T|}(t)<\infty .
$$

Hence, $\tau\left(E_{|T|}(\{0\})\right)=\mu_{|T|}(\{0\})=0$, so that $\operatorname{ker}(T)=\{0\}$. Since $\mathcal{M}$ is finite, also $\operatorname{ker}\left(T^{*}\right)=\{0\}$, which implies that $T$ has a closed, densely defined inverse $T^{-1} \in \tilde{\mathscr{M}}$. Take a unitary $U \in \mathscr{M}$ such that $T=U|T|$. Then

$$
\left|T^{-1}\right|=U|T|^{-1} U^{*} .
$$


Hence, $\mu_{\left|T^{-1}\right|}=\mu_{|T|^{-1}}$. Since $\mu_{|T|^{-1}}$ is the push-forward measure of $\mu_{|T|}$ via the map $t \mapsto \frac{1}{t}$, we now have that

$$
\begin{aligned}
\int_{1}^{\infty} \log t d \mu_{\left|T^{-1}\right|}(t) & =\int_{1}^{\infty} \log t d \mu_{|T|^{-1}}(t)=\int_{0}^{1} \log \left(\frac{1}{t}\right) d \mu_{|T|}(t) \\
& =-\int_{0}^{1} \log t d \mu_{|T|}(t)<\infty
\end{aligned}
$$

Hence, $T^{-1} \in \mathcal{M}^{\Delta}$ and

$$
\log \Delta\left(T^{-1}\right)=\int_{0}^{\infty} \log \left(\frac{1}{t}\right) d \mu_{|T|}(t)=-\log \Delta(T),
$$

i.e. $\Delta\left(T^{-1}\right)=\frac{1}{\Delta(T)}$.

Lemma 2.4. Let $T \in \tilde{M}$. Then the following are equivalent:

(a) $T \in \mathcal{M}^{\Delta}$, i.e. $\int_{0}^{\infty} \log ^{+}(t) d \mu_{|T|}(t)<\infty$.

(b) $T=A B^{-1}$ for some $A, B \in \mathscr{M}$ with $\Delta(B)>0$.

(c) $T=C^{-1} D$ for some $C, D \in \mathscr{M}$ with $\Delta(C)>0$.

Moreover, if $T \in \mathcal{M}^{\Delta}$ and $T=A B^{-1}=C^{-1} D$ for some $A, B, C, D \in \mathscr{M}$ with $\Delta(B), \Delta(C)>0$, then

$$
\Delta(T)=\frac{\Delta(A)}{\Delta(B)}=\frac{\Delta(D)}{\Delta(C)} .
$$

Proof. If $T \in \mathcal{M}^{\Delta}$, then $T=U|T|$ for some unitary $U \in \mathcal{M}$, and $T=$ $A B^{-1}$, where

$$
A=U|T|\left(|T|^{2}+\mathbf{1}\right)^{-\frac{1}{2}} \in \mathscr{M}
$$

and

$$
B=\left(|T|^{2}+\mathbf{1}\right)^{-\frac{1}{2}} \in \mathcal{M} .
$$

Since $\frac{1}{2} \log \left(t^{2}+1\right) \leq \log (2 t)$ when $t \geq 1$, we get that

$$
\begin{aligned}
\log \Delta(B) & =-\frac{1}{2} \int_{0}^{\infty} \log \left(t^{2}+1\right) d \mu_{|T|}(t) \\
& \geq-\frac{1}{2} \int_{[0,1[} \log 2 d \mu_{|T|}(t)-\int_{[1, \infty[} \log (2 t) d \mu_{|T|}(t)>-\infty,
\end{aligned}
$$

that is, $\Delta(B)>0$. 
Also, $T=U|T| U^{*} U$, and with

$$
\begin{gathered}
S=U|T| U^{*}, \\
C=\left(S^{2}+\mathbf{1}\right)^{-\frac{1}{2}} \in \mathcal{M},
\end{gathered}
$$

and

$$
D=S\left(S^{2}+\mathbf{1}\right)^{-\frac{1}{2}} \in \mathcal{M},
$$

we have that $T=C^{-1} D U$. Moreover,

$$
\begin{aligned}
\log \Delta(C) & =-\frac{1}{2} \int_{0}^{\infty} \log \left(t^{2}+1\right) d \mu_{S}(t) \\
& =-\frac{1}{2} \int_{0}^{\infty} \log \left(t^{2}+1\right) d \mu_{|T|}(t)>-\infty
\end{aligned}
$$

i.e. $\Delta(C)>0$.

Now we have shown that (a) implies (b) and (c). On the other hand, if $T=A B^{-1}$ for some $A, B \in \mathcal{M}$ with $\Delta(B)>0$, then we may assume that $B \geq 0$. Then

$$
\tau\left(\log ^{+}|T|\right) \leq \tau\left(\log \left(\mathbf{1}+|T|^{2}\right)\right)=\tau\left(\log \left(\mathbf{1}+B^{-1} A^{*} A B^{-1}\right)\right) .
$$

Since $B^{-1} A^{*} A B^{-1} \leq\|A\|^{2} B^{-2}$, and since $t \mapsto \log (1+t)$ is operator monotone on $[0, \infty)$, we get that

$$
\begin{aligned}
\tau\left(\log ^{+}|T|\right) & \leq \tau\left(\log \left(\mathbf{1}+\|A\|^{2} B^{-2}\right)\right) \\
& \leq \tau\left(\log \left(\left(1+\|A\|^{2}\right)\left(\mathbf{1}+B^{-2}\right)\right)\right) \\
& =\log \left(1+\|A\|^{2}\right)+\tau\left(\log \left(\mathbf{1}+B^{-2}\right)\right) .
\end{aligned}
$$

Since $B$ is bounded and $\Delta(B)>0$,

$$
\begin{aligned}
\tau\left(\log \left(\mathbf{1}+B^{-2}\right)\right) & =\tau\left(\log \left(B^{2}+\mathbf{1}\right)\right)-2 \tau(\log B) \\
& \leq \log \left(\|B\|^{2}+1\right)-2 \Delta(B) \\
& <\infty .
\end{aligned}
$$

This shows that $T \in \mathscr{M}^{\Delta}$, i.e. (b) implies (a). It follows that if $T=C^{-1} D$ for some $C, D \in \mathscr{M}$ with $\Delta(C)>0$, then $T^{*} \in \mathcal{M}^{\Delta}$. Take a unitary $U \in \mathcal{M}$ such that $T=U|T|$. Then $\left|T^{*}\right|=U|T| U^{*}$, implying that $\mu_{\left|T^{*}\right|}=\mu_{|T|}$. Hence $T$ belongs to $\mathscr{M}^{\Delta}$ as well, and (c) implies (a). 
Now, let $T \in \mathcal{M}^{\Delta}$. Then $T=A B^{-1}=C^{-1} D$ for some $A, B, C, D \in \mathcal{M}$ with $\Delta(B), \Delta(C)>0$. Moreover, for all such choices of $A, B, C$ and $D$,

$$
C A=C\left(A B^{-1}\right) B=C\left(C^{-1} D\right) B=D B .
$$

Since $\Delta$ is multiplicative on $\mathscr{M}$ (cf. [7]), it follows that

$$
\Delta(C) \Delta(A)=\Delta(C A)=\Delta(D B)=\Delta(D) \Delta(B) .
$$

Hence,

$$
\frac{\Delta(A)}{\Delta(B)}=\frac{\Delta(D)}{\Delta(C)}
$$

In particular, with $A$ and $B$ as in (2.6) and (2.7), respectively, we have that $\Delta(B)>0, T=A B^{-1}$, and

$$
\log \Delta(A)=\int_{0}^{\infty} \log \left(\frac{t}{\sqrt{t^{2}+1}}\right) d \mu_{|T|}(t),
$$

and

$$
\log \Delta(B)=\int_{0}^{\infty} \log \left(\frac{1}{\sqrt{t^{2}+1}}\right) d \mu_{|T|}(t)
$$

so that

$$
\log \Delta(T)=\log \Delta(A)-\log \Delta(B) .
$$

Then by (2.12), for all choices of $C, D \in \mathscr{M}$ with $\Delta(C)>0$ and $T=C^{-1} D$,

$$
\frac{\Delta(D)}{\Delta(C)}=\frac{\Delta(A)}{\Delta(B)}=\Delta(T) .
$$

Then finally, by (2.12), for all choices of $A, B \in \mathcal{M}$ with $\Delta(B)>0$ and $T=A B^{-1}$, we also have that

$$
\frac{\Delta(A)}{\Delta(B)}=\Delta(T)
$$

Proposition 2.5. If $S, T \in \mathcal{M}^{\Delta}$, then $S T \in \mathcal{M}^{\Delta}$, and

$$
\Delta(S T)=\Delta(S) \Delta(T) .
$$

Proof. Let $S, T \in \mathcal{M}^{\Delta}$. Take $A, B, C, D \in \mathcal{M}$ with $\Delta(B), \Delta(C)>0$, such that $T=A B^{-1}$ and $S=C^{-1} D$. Then

$$
S T=C^{-1} D A B^{-1},
$$


where $D A B^{-1} \in \mathscr{M}^{\Delta}$. Hence there exist $E, F \in \mathscr{M}$ with $\Delta(E)>0$ such that $D A B^{-1}=E^{-1} F$. It follows that

$$
S T=C^{-1} E^{-1} F=(E C)^{-1} F,
$$

where $E C, F \in \mathscr{M}$, and $\Delta(E C)=\Delta(E) \Delta(C)>0$. That is, $S T$ belongs to $M^{\Delta}$.

To prove (2.13), we let $A, B, C, D, E, F$ be as above. Applying (2.5) to $S T=(E C)^{-1} F, S=C^{-1} D$ and $T=A B^{-1}$, we get that

$$
\begin{aligned}
\Delta(S T) & =\frac{\Delta(F)}{\Delta(E C)}=\frac{\Delta(F)}{\Delta(E) \Delta(C)}=\frac{\Delta(D A)}{\Delta(B)} \frac{1}{\Delta(C)} \\
& =\frac{\Delta(A)}{\Delta(B)} \frac{\Delta(D)}{\Delta(C)}=\Delta(S) \Delta(T) .
\end{aligned}
$$

Proposition 2.6. $\mathscr{M}^{\Delta}$ is a subspace of $\tilde{\mathscr{M}}$. In particular, for $T \in \mathscr{M}^{\Delta}$ and $\lambda \in \mathrm{C}, T-\lambda \mathbf{1} \in \mathscr{M}^{\Delta}$.

Proof. Clearly, if $T \in \mathcal{M}^{\Delta}$ and $\alpha \in \mathrm{C}$, then $\alpha T \in \mathcal{M}^{\Delta}$. If $S, T \in \mathcal{M}$, choose $A, B, C, D \in \mathscr{M}$ with $\Delta(B)>0, \Delta(C)>0$ and such that

$$
S=C^{-1} D, \quad T=A B^{-1} .
$$

Then

$$
S+T=C^{-1}(D B+C A) B^{-1},
$$

where $D B+C A \in \mathcal{M}$ and $B^{-1}, C^{-1} \in \mathscr{M}^{\Delta}$ (cf. Lemma 2.3). Then, by Proposition 2.5, $S+T \in \mathcal{M}^{\Delta}$.

In the following we consider a fixed operator $T \in \mathcal{M}^{\Delta}$. Then we define $f: \mathrm{C} \rightarrow[-\infty, \infty)$ by

$$
f(\lambda)=L(T-\lambda \mathbf{1}):=\log \Delta(T-\lambda \mathbf{1}), \quad(\lambda \in \mathrm{C}) .
$$

The next thing we want to prove is:

TheORem 2.7. $f$ given by (2.15) is subharmonic in $\mathrm{C}$, and

$$
d \mu_{T}=\frac{1}{2 \pi} \nabla^{2} f d \lambda
$$

(taken in the distribution sense) defines a probability measure on (C, $\left.B_{2}\right) . \mu_{T}$ is the unique probability measure on $\left(\mathrm{C}, \mathrm{B}_{2}\right)$ satisfying

(i)

$$
\int_{\mathrm{C}} \log ^{+}|z| d \mu_{T}(z)<\infty
$$


(ii)

$$
\forall \lambda \in \mathrm{C}: \quad L(T-\lambda \mathbf{1})=\int_{\mathrm{C}} \log |\lambda-z| d \mu_{T}(z)
$$

Moreover,

(iii)

$$
\int_{\mathrm{C}} \log ^{+}|z| d \mu_{T}(z)=\frac{1}{2 \pi} \int_{0}^{2 \pi} f\left(e^{i \theta}\right) d \theta
$$

The following lemma was proven by F. Larsen in his unpublished thesis (cf. [14, section 2]). For the convenience of the reader we include a (somewhat different) proof.

Lemma 2.8. Let $a, b \in \mathcal{M}$ and let $\varepsilon>0$. Define $g_{\varepsilon}, g: \mathrm{C} \rightarrow \mathrm{R}$ by

$$
g_{\varepsilon}(\lambda)=\frac{1}{2} \tau\left(\log \left((a-\lambda b)^{*}(a-\lambda b)+\varepsilon \mathbf{1}\right)\right),
$$

and

$$
g(\lambda)=\log \Delta(a-\lambda b) .
$$

Then $g_{\varepsilon}$ is subharmonic, and if $g(\lambda)>-\infty$ for some $\lambda \in \mathrm{C}$, then $g$ is subharmonic as well.

Proof. Let $\lambda_{1}=\operatorname{Re}(\lambda), \lambda_{2}=\operatorname{Im}(\lambda), \lambda \in \mathrm{C}$. At first we show that $\left(\lambda_{1}, \lambda_{2}\right) \mapsto g_{\varepsilon}\left(\lambda_{1}+i \lambda_{2}\right)$ is a $C^{2}$-function in $\mathrm{R}^{2}$. Fix $\varepsilon>0$, and define $h, k: \mathrm{C} \rightarrow \mathcal{M}$ by

$$
\begin{aligned}
& h(\lambda)=(a-\lambda b)^{*}(a-\lambda b)+\varepsilon \mathbf{1}, \\
& k(\lambda)=(a-\lambda b)(a-\lambda b)^{*}+\varepsilon \mathbf{1} .
\end{aligned}
$$

Then $h$ and $k$ are second order polynomials in $\left(\lambda_{1}, \lambda_{2}\right)$ with coefficients in $\mathcal{M}$, and $h(\lambda) \geq \varepsilon \mathbf{1}, k(\lambda) \geq \varepsilon \mathbf{1}$ for all $\lambda \in \mathrm{C}$. Hence, by [11, Lemma 4.6],

$$
g_{\varepsilon}(\lambda)=\frac{1}{2} \tau(\log h(\lambda)), \quad \lambda \in \mathrm{C},
$$

has continuous partial derivatives given by

$$
\frac{\partial g_{\varepsilon}}{\partial \lambda_{j}}=\frac{1}{2} \tau\left(h^{-1} \frac{\partial h}{\partial \lambda_{j}}\right), \quad j=1,2 .
$$

Therefore, by [11, Lemma 3.2], $g_{\varepsilon}$ is a $C^{2}$-function with (2.19)

$$
\frac{\partial^{2} g_{\varepsilon}}{\partial \lambda_{i} \partial \lambda_{j}}=\frac{1}{2} \tau\left(-h^{-1} \frac{\partial h}{\partial \lambda_{i}} h^{-1} \frac{\partial h}{\partial \lambda_{j}}+h^{-1} \frac{\partial^{2} h}{\partial \lambda_{i} \partial \lambda_{j}}\right), \quad i=1,2, \quad j=1,2 .
$$


Since $g_{\varepsilon}$ is $C^{2}, g_{\varepsilon}$ is subharmonic if and only if its Laplacian

$$
\frac{\partial^{2} g_{\varepsilon}}{\partial \lambda_{1}^{2}}+\frac{\partial^{2} g_{\varepsilon}}{\partial \lambda_{2}^{2}}
$$

is positive. Following standard notation, we let

$$
\frac{\partial}{\partial \lambda}=\frac{1}{2}\left(\frac{\partial}{\partial \lambda_{1}}-i \frac{\partial}{\partial \lambda_{2}}\right) \quad \text { and } \quad \frac{\partial}{\partial \bar{\lambda}}=\frac{1}{2}\left(\frac{\partial}{\partial \lambda_{1}}+i \frac{\partial}{\partial \lambda_{2}}\right) .
$$

Then

$$
\frac{\partial^{2} g_{\varepsilon}}{\partial \lambda_{1}^{2}}+\frac{\partial^{2} g_{\varepsilon}}{\partial \lambda_{2}^{2}}=4 \frac{\partial^{2} g_{\varepsilon}}{\partial \bar{\lambda} \partial \lambda}
$$

By application of (2.19), we find that

$$
\frac{\partial^{2} g_{\varepsilon}}{\partial \bar{\lambda} \partial \lambda}=\frac{1}{2} \tau\left(-h^{-1} \frac{\partial h}{\partial \bar{\lambda}} h^{-1} \frac{\partial h}{\partial \lambda}+h^{-1} \frac{\partial^{2} h}{\partial \bar{\lambda} \partial \lambda}\right) .
$$

Since

$$
h(\lambda)=a^{*} a-\lambda a^{*} b-\bar{\lambda} b^{*} a+|\lambda|^{2} b^{*} b+\varepsilon \mathbf{1},
$$

we have

$$
\begin{aligned}
& \frac{\partial h}{\partial \lambda}=-a^{*} b+\bar{\lambda} b^{*} b=-(a-\lambda b)^{*} b, \\
& \frac{\partial h}{\partial \bar{\lambda}}=-b^{*} a+\lambda b^{*} b=-b^{*}(a-\lambda b),
\end{aligned}
$$

and

$$
\frac{\partial^{2} h}{\partial \bar{\lambda} \partial \lambda}=b^{*} b
$$

Applying the identity $x\left(x^{*} x+\varepsilon \mathbf{1}\right)^{-1}=\left(x x^{*}+\varepsilon \mathbf{1}\right)^{-1} x$ to $x=a-\lambda b$, we find that

$$
\begin{aligned}
\frac{\partial^{2} h}{\partial \bar{\lambda} \partial \lambda}-\frac{\partial h}{\partial \bar{\lambda}} h^{-1} \frac{\partial h}{\partial \lambda} & =b^{*} b-b^{*} x\left(x^{*} x+\varepsilon \mathbf{1}\right)^{-1} x^{*} b \\
& =b^{*} b-b^{*}\left(x x^{*}+\varepsilon \mathbf{1}\right)^{-1} x x^{*} b \\
& =b^{*} b-b^{*}\left(\mathbf{1}-\varepsilon\left(x x^{*}+\varepsilon \mathbf{1}\right)^{-1}\right) b \\
& =\varepsilon b^{*}\left(x x^{*}+\varepsilon \mathbf{1}\right)^{-1} b \\
& =\varepsilon b^{*} k^{-1} b .
\end{aligned}
$$


Then by (2.20),

$$
\begin{aligned}
\frac{\partial^{2} g_{\varepsilon}}{\partial \bar{\lambda} \partial \lambda} & =\frac{\varepsilon}{2} \tau\left(h(\lambda)^{-1} b^{*} k(\lambda)^{-1} b\right) \\
& =\frac{\varepsilon}{2} \tau\left(h(\lambda)^{-\frac{1}{2}} b^{*} k(\lambda)^{-1} b h(\lambda)^{-\frac{1}{2}}\right) \geq 0,
\end{aligned}
$$

showing that $g_{\varepsilon}$ is subharmonic.

Fix $\lambda \in \mathrm{C}$, and let $x=a-\lambda b$ as above. Then

$$
g_{\varepsilon}(\lambda)=\frac{1}{2} \int_{0}^{\|x\|} \log \left(t^{2}+\varepsilon\right) d \mu_{|x|}(t),
$$

and

$$
g(\lambda)=\frac{1}{2} \int_{0}^{\|x\|} \log \left(t^{2}\right) d \mu_{|x|}(t) .
$$

Hence, $g_{\varepsilon}$ is a monotonically decreasing function of $\varepsilon>0$, and

$$
g(\lambda)=\lim _{\varepsilon \rightarrow 0^{+}} g_{\varepsilon}(\lambda) .
$$

According to [13], $g$ is then either subharmonic or identically $-\infty$.

Proposition 2.9. Let $T \in \mathcal{M}^{\Delta}$. Then the function $f: C \rightarrow[-\infty, \infty[$ given by

$$
f(\lambda)=\log \Delta(T-\lambda \mathbf{1})
$$

is subharmonic in $\mathrm{C}$.

Proof. Define $T_{1}, T_{2} \in \mathscr{M}$ by

$$
T_{1}=T\left(T^{*} T+\mathbf{1}\right)^{-\frac{1}{2}}
$$

and

$$
T_{2}=\left(T^{*} T+\mathbf{1}\right)^{-\frac{1}{2}}
$$

Then for every $\lambda \in C$,

$$
T-\lambda \mathbf{1}=\left(T_{1}-\lambda T_{2}\right) T_{2}^{-1},
$$

where $\Delta\left(T_{2}\right)>0$ (cf. (2.8)). Thus, $T-\lambda \mathbf{1} \in \mathcal{M}^{\Delta}$ with

$$
\Delta(T-\lambda \mathbf{1})=\Delta\left(T_{1}-\lambda T_{2}\right) \Delta\left(T_{2}\right)^{-1},
$$

i.e.

$$
f(\lambda)=L(T-\lambda \mathbf{1})=L\left(T_{1}-\lambda T_{2}\right)-L\left(T_{2}\right) .
$$


Then by Lemma $2.8, f$ is either subharmonic or identically $-\infty$. With

$$
h(\lambda)=L\left(T_{2}-\lambda T_{1}\right)-L\left(T_{2}\right),
$$

$h(0)=0>-\infty$, and it follows from Lemma 2.8 that $h$ is subharmonic. In particular, $h(\lambda)>-\infty$ for almost every $\lambda \in \mathrm{C}$ w.r.t. Lebesgue measure. For $\lambda \in \mathrm{C} \backslash\{0\}$,

$$
f(\lambda)=h\left(\frac{1}{\lambda}\right)+\log |\lambda| .
$$

Hence, $f$ is not identically $-\infty$.

Recall from [13, Section 3.5.4] that one can associate to every subharmonic function $u$ the socalled Riesz measure $\mu_{u}$, which is a positive Borel measure on $\mathrm{R}^{2}$ uniquely determined by

$$
\forall \phi \in C_{c}^{\infty}\left(\mathrm{R}^{2}\right): \quad \frac{1}{2 \pi} \int_{\mathrm{R}^{2}} u \nabla^{2} \phi d m=\int_{\mathrm{R}^{2}} \phi d \mu_{u} .
$$

One uses the notation $d \mu_{u}=\frac{1}{2 \pi} \nabla^{2} u d \lambda$, and this is what is meant by (2.16).

In order to prove the rest of Theorem 2.7, we need some general lemmas on subharmonic functions:

Lemma 2.10. Let $g: \mathrm{C} \rightarrow[-\infty, \infty[$ be a subharmonic function, and for $r>0$ define

$$
\begin{aligned}
& m(g, r)=\frac{1}{2 \pi} \int_{0}^{2 \pi} g\left(r e^{i \theta}\right) d \theta, \\
& M(g, r)=\sup _{|z|=r} g(z) .
\end{aligned}
$$

Then

$$
g(0)=\lim _{r \rightarrow 0} m(g, r)=\lim _{r \rightarrow 0} M(g, r) .
$$

Proof. Clearly, $m(g, r) \leq M(g, r)$ for every $r>0$. Moreover, since $g$ is subharmonic, $g(0) \leq m(g, r),(r>0)$. It follows that

$$
g(0) \leq\left\{\begin{array}{l}
\lim \sup _{r \rightarrow 0} m(g, r) \leq \limsup _{r \rightarrow 0} M(g, r) \\
\liminf _{r \rightarrow 0} m(g, r) \leq \liminf _{r \rightarrow 0} M(g, r)
\end{array}\right.
$$

Now, every upper semicontinuous function attains a maximum on every compact set. In particular, there exists for every $r>0$ a complex number $z_{r}$ of modulus $r$ such that $g\left(z_{r}\right)=M(g, r) . z_{r} \rightarrow 0$ as $r \rightarrow 0$, and therefore

$$
g(0) \geq \limsup _{r \rightarrow 0} g\left(z_{r}\right)=\limsup _{r \rightarrow 0} M(g, r) .
$$


It follows from (2.28) and (2.29) that

$$
g(0) \leq \liminf _{r \rightarrow 0} m(g, r) \leq\left\{\begin{array}{c}
\limsup _{r \rightarrow 0} m(g, r) \\
\liminf _{r \rightarrow 0} M(g, r)
\end{array}\right\} \leq \limsup _{r \rightarrow 0} M(g, r) \leq g(0)
$$

so the four inequalities above are in fact identities, and this proves (2.27).

LEMMA 2.11. $f$ given by (2.15) satisfies

$$
\lim _{r \rightarrow \infty}(M(f, r)-\log r)=\lim _{r \rightarrow \infty}(m(f, r)-\log r)=0 .
$$

Proof. Define $h: \mathrm{C} \rightarrow[-\infty, \infty[$ by

$$
h(\lambda)=L\left(T_{2}-\lambda T_{1}\right)-L\left(T_{2}\right), \quad \lambda \in \mathrm{C} .
$$

Then $h$ is subharmonic with $h(0)=0$, and it follows from Lemma 2.10 that

$$
0=\lim _{r \rightarrow 0} m(h, r)=\lim _{r \rightarrow 0} M(h, r) .
$$

Since

$$
h(\lambda)=\log |\lambda|+f\left(\frac{1}{\lambda}\right), \quad \lambda \neq 0,
$$

we get that when $r>0$,

$$
\begin{gathered}
M(f, r)=M\left(h, \frac{1}{r}\right)+\log r, \\
m(f, r)=m\left(h, \frac{1}{r}\right)+\log r,
\end{gathered}
$$

and combining this with (2.32) we obtain the desired result.

Lemma 2.12. Let $R>r>0$, and let $g$ be subharmonic in $\mathrm{C}$. Then with $d \mu=\frac{1}{2 \pi} \nabla^{2} g d \lambda$ and

$$
\psi(z)= \begin{cases}\log \left(\frac{R}{r}\right), & |z| \leq r \\ \log \left(\frac{R}{|z|}\right), & r<|z|<R \\ 0, & |z| \geq R\end{cases}
$$

one has that

$$
m(g, R)-m(g, r)=\int_{C} \psi(z) d \mu(z) .
$$

Proof. Cf. [13, (3.5.7)]. 
Proof of Theorem 2.7. When $R>1>0$ define $\psi_{R}: \mathrm{C} \rightarrow \mathrm{R}$ by

$$
\psi_{R}(z)= \begin{cases}\log R, & |z| \leq 1 \\ \log \left(\frac{R}{|z|}\right), & 1<|z|<R \\ 0, & |z| \geq R\end{cases}
$$

Then, according to Lemma 2.12,

$$
\int_{C} \psi_{R}(z) d \mu_{T}(z)=m(f, R)-m(f, 1) .
$$

Now, $\frac{1}{\log R} \psi_{R} \nearrow 1$ as $R \rightarrow \infty$, so by the Monotone Convergence Theorem, (2.35) and Lemma 2.11,

$$
\mu_{T}(\mathrm{C})=\lim _{R \rightarrow \infty} \frac{m(f, R)-m(f, 1)}{\log R}=1,
$$

that is, $\mu_{T}$ is a probability measure.

When $R>1$, let

$$
\omega_{R}(z)=\log R-\psi_{R}(z), \quad z \in \mathrm{C} .
$$

Then $\omega_{R}(z) \nearrow \log ^{+}|z|$ as $R \rightarrow \infty$, and hence by one more application of Lemma 2.11,

$$
\begin{aligned}
\int_{C} \log ^{+}|z| d \mu_{T}(z)=\lim _{R \rightarrow \infty} \int_{C} \omega_{R} d \mu_{T} & =\lim _{R \rightarrow \infty}(\log R-m(f, R)+m(f, 1)) \\
& =m(f, 1),
\end{aligned}
$$

proving (2.18). Note that since $f$ is subharmonic, (2.18) imlies that $\int_{\mathrm{C}} \log ^{+}|z| d \mu_{T}(z)<\infty$.

To see that (2.17) holds, it suffices to consider the case $\lambda=0$. Indeed, for fixed $\lambda \in C$ one easily sees that $\mu_{T-\lambda 1}$ is the push-forward measure of $\mu_{T}$ under the map $z \mapsto z-\lambda$ (cf. Lemma 2.14), and therefore

$$
\int_{C} \log |z-\lambda| d \mu_{T}(z)=\int_{C} \log |z| d \mu_{T-\lambda \mathbf{1}}(z) .
$$

In the case $\lambda=0$ one has to compute the integrals $\int_{C} \log ^{ \pm}|z| d \mu_{T}(z)$. We have just seen that

$$
\int_{C} \log ^{+}|z| d \mu_{T}(z)=m(f, 1)
$$


and with

$$
\chi_{r}(z)= \begin{cases}\log \frac{1}{r}, & |z| \leq r \\ \log \frac{1}{|z|}, & r<|z| \leq 1 \\ 0, & |z| \geq 1\end{cases}
$$

$\chi_{r}(z) \nearrow \log ^{-}|z|$ as $r \searrow 0$. Hence by Lemma 2.10 and Lemma 2.12,

$$
\begin{aligned}
\int_{C} \log ^{-}|z| d \mu_{T}(z)=\lim _{r \rightarrow 0} \int_{C} \chi_{r} d \mu_{T} & =\lim _{r \rightarrow 0}(m(f, 1)-m(f, r)) \\
& =m(f, 1)-f(0) .
\end{aligned}
$$

Combining this with (2.38) we get that

$$
\int_{C} \log |z| d \mu_{T}(z)=f(0)=L(T),
$$

as desired.

In order to prove that $\mu_{T}$ is uniquely determined by (i) and (ii) of Theorem 2.7, suppose $v \in \operatorname{Prob}(C)$ satisfies

$$
\int_{C} \log ^{+}|z| d v(z)<\infty
$$

and

$$
\forall \lambda \in \mathrm{C}: \quad \int_{\mathrm{C}} \log |z-\lambda| d \nu(z)=L(T-\lambda \mathbf{1}) .
$$

Note that (2.39) implies that $\int_{\mathrm{C}} \log |z-\lambda| d \nu(z)$ is well-defined, since

$$
\log |z-\lambda| \leq \log (|z|+|\lambda|)
$$

and

$$
|z|+|\lambda| \leq(|\lambda|+1) \cdot \max \{1,|z|\}
$$

Hence

$$
\log |z-\lambda| \leq \log (|\lambda|+1)+\log ^{+}|z|
$$

Since $\mu$ and $\nu$ are both probability measures, it follows from a $C^{\infty}$-version of Urysohn's Lemma (cf. [8, (8.18)]) that if

$$
\int_{C} \phi d \mu_{T}=\int_{C} \phi d v
$$


for every function $\phi \in C_{c}^{\infty}\left(\mathrm{R}^{2}\right)$, then $\mu_{T}=\nu$. Then consider an arbitrary function $\phi \in C_{c}^{\infty}\left(\mathrm{R}^{2}\right)$. Since the Laplacian of $w \mapsto \frac{1}{2 \pi} \log |w-z|$ (in the distribution sense) is the Dirac measure $\delta_{z}$ at $z$, one has that

$$
\begin{aligned}
\int_{C} \phi(z) d \nu(z) & =\int_{C}\left(\int_{C} \phi(\lambda) \delta_{z}(\lambda)\right) d v(z) \\
& =\frac{1}{2 \pi} \int_{C}\left(\int_{C}\left(\nabla^{2} \phi\right)(\lambda) \log |z-\lambda| d \lambda\right) d \nu(z) .
\end{aligned}
$$

At this place we would like to reverse the order of integration, but it is not entirely clear that this is a legal operation. Therefore we put $M=\left\|\nabla^{2} \phi\right\|_{\infty}$, and take $\chi \in C_{c}^{\infty}\left(\mathrm{R}^{2}\right)$ such that $0 \leq \chi \leq 1$ and $\chi_{\left.\right|_{\text {supp }\left(\nabla^{2} \phi\right)}}=1$. With

$$
\psi_{1}=\frac{1}{2}\left(M+\nabla^{2} \phi\right) \chi
$$

and

$$
\psi_{2}=\frac{1}{2}\left(M-\nabla^{2} \phi\right) \chi
$$

one has that $\psi_{1}, \psi_{2} \in C_{c}^{\infty}\left(\mathbf{R}^{2}\right)^{+}$, and $\nabla^{2} \phi=\psi_{1}-\psi_{2}$.

Also not that, according to (2.41),

$$
h(\lambda, z):=\log (|\lambda|+1)+\log ^{+}|z|-\log |z-\lambda| \geq 0 .
$$

Therefore by Tonelli's Theorem

$$
\int_{\mathrm{C}} \psi_{i}(\lambda) \int_{\mathrm{C}} h(\lambda, z) d \nu(z) d \lambda=\int_{\mathrm{C}} \int_{\mathrm{C}} \psi_{i}(\lambda) h(\lambda, z) d \lambda d \nu(z), \quad i=1,2 .
$$

The map $\lambda \mapsto L(T-\lambda \mathbf{1})$ is subharmonic and therefore locally integrable. Since

$$
\int_{C} h(\lambda, z) d v(z)=\log (|\lambda|+1)+\int_{C} \log ^{+}|z| d \nu(z)-L(T-\lambda \mathbf{1}),
$$

where $\lambda \mapsto L(T-\lambda \mathbf{1})$ is subharmonic and therefore locally integrable,

$$
\int_{\mathrm{C}} \psi_{i}(\lambda) \int_{\mathrm{C}} h(\lambda, z) d \nu(z) d \lambda<\infty, \quad i=1,2 .
$$

It now follows from (2.43) that

$$
\int_{C}\left(\nabla^{2} \phi\right)(\lambda) \int_{C} h(\lambda, z) d v(z) d \lambda=\int_{C} \int_{C}\left(\nabla^{2} \phi\right)(\lambda) h(\lambda, z) d \lambda d v(z),
$$


and since

$$
\int_{C}\left|\left(\nabla^{2} \phi\right)(\lambda)\right| \int_{C} \log (|\lambda|+1) d v(z) d \lambda<\infty,
$$

and

$$
\int_{C}\left|\left(\nabla^{2} \phi\right)(\lambda)\right| \int_{C} \log ^{+}|z| d \nu(z) d \lambda<\infty,
$$

we deduce that

$$
\begin{aligned}
\int_{C} \phi(z) d \nu(z) & =\frac{1}{2 \pi} \int_{C}\left(\int_{C}\left(\nabla^{2} \phi\right)(\lambda) \log |\lambda-z| d \lambda\right) d \nu(z) \\
& =\frac{1}{2 \pi} \int_{C}\left(\nabla^{2} \phi\right)(\lambda) \int_{C} \log |\lambda-z| d \nu(z) d \lambda \\
& =\frac{1}{2 \pi} \int_{C}\left(\nabla^{2} \phi\right)(\lambda) L(T-\lambda \mathbf{1}) d \lambda \\
& =\int_{C} \phi(z) d \mu_{T}(z),
\end{aligned}
$$

and this is the desired identity.

It follows from Theorem 2.7 that one can associate to every operator $T \in$ $\mathcal{M}^{\Delta}$ a probability measure $\mu_{T}$ on $\left(\mathrm{C}, \mathrm{B}_{2}\right)$, such that in the case where $T \in \mathcal{M}$, $\mu_{T}$ agrees with the Brown measure of $T$. Therefore we make the following definition:

DEFInition 2.13. For $T \in \mathcal{M}^{\Delta}$ we shall say that the probability measure $\mu_{T}$ from Theorem 2.7 is the Brown measure of $T$.

In the remaining part of this section we will see that many of the properties of the Brown measure for bounded operators carry over to this more general setting.

Proposition 2.14. Let $T \in \mathcal{M}^{\Delta}$. Then for every $r>0$ and every $\lambda \in \mathrm{C}$, the Brown measure of $r T+\lambda \mathbf{1}, \mu_{r T+\lambda \mathbf{1}}$, is the push-forward measure of $\mu_{T}$ via the map $z \mapsto r z+\lambda$.

Proof. Making use of Urysohn's Lemma for $C^{\infty}$-functions on $\mathrm{R}^{2}$ (cf. [8, (8.18)]) and the fact that both of the measures considered here are probability measures, one easily sees that if

$$
\int_{C} \phi(z) d \mu_{r T+\lambda \mathbf{1}}(z)=\int_{C} \phi(r z+\lambda) d \mu_{T}(z)
$$

for every $\phi \in C_{c}^{\infty}\left(\mathrm{R}^{2}\right)$, then the two measures in speak agree on compact sets and hence on all of $B_{2}$. 
Let $\phi \in C_{c}^{\infty}\left(\mathrm{R}^{2}\right)$. Then by definition,

$$
\begin{aligned}
\int_{C} \phi(r z+\lambda) d \mu_{T}(z) & =\frac{1}{2 \pi} \int_{C}\left(\frac{\partial^{2}}{\partial z_{1}^{2}}+\frac{\partial^{2}}{\partial z_{2}^{2}}\right) \phi(r z+\lambda) f(z) d z \\
& =\frac{1}{2 \pi} \int_{C} r^{2}\left(\frac{\partial^{2}}{\partial w_{1}^{2}}+\frac{\partial^{2}}{\partial w_{2}^{2}}\right) \phi(w) f\left(\frac{1}{r}(w-\lambda)\right) \frac{1}{r^{2}} d w \\
& =\frac{1}{2 \pi} \int_{C} \nabla^{2} \phi(w) f\left(\frac{1}{r}(w-\lambda)\right) d w \\
& =\frac{1}{2 \pi} \int_{C} \nabla^{2} \phi(w)[L(r T+\lambda \mathbf{1}-w \mathbf{1})-\log r] d w \\
& =\int_{C} \phi(w) d \mu_{r T+\lambda \mathbf{1}}(w)-\log r \cdot \int_{C} \nabla^{2} \phi(w) d w \\
& =\int_{C} \phi(w) d \mu_{r T+\lambda \mathbf{1}}(w),
\end{aligned}
$$

where the last identity follows from Green's Theorem.

Proposition 2.15. For every $T \in \mathcal{M}^{\Delta}$ and every $m \in \mathrm{N}, \mu_{T^{m}}$ is the pushforward measure of $\mu_{T}$ via the map $z \mapsto z^{m}$.

Proof. Let $v \in \operatorname{Prob}(\mathrm{C})$ denote the push-forward measure of $\mu_{T}$ under the map $z \mapsto z^{m}$. According to Theorem 2.7 it suffices to prove that

$$
\int_{\mathrm{C}} \log ^{+}|z| d v(z)<\infty
$$

and

$$
\forall \lambda \in \mathrm{C}: \quad \int_{\mathrm{C}} \log |\lambda-z| d \nu(z)=L\left(T^{m}-\lambda \mathbf{1}\right) .
$$

Here

$$
\int_{\mathrm{C}} \log ^{+}|z| d \nu(z)=\int_{\mathrm{C}} \log ^{+}\left|z^{m}\right| d \mu_{T}(z)=m \int_{\mathrm{C}} \log ^{+}|z| d \mu_{T}(z)<\infty,
$$

and if we let $\theta_{1}, \ldots, \theta_{m}$ denote the $m$ complex roots of $Q(z)=z^{m}-1$, then for every $\lambda \in \mathrm{C}$,

$$
\left|\lambda-z^{m}\right|=\prod_{k=1}^{m}\left|\theta_{k} \lambda^{\frac{1}{m}}-z\right|
$$


Hence

$$
\begin{aligned}
\int_{C} \log |\lambda-z| d \nu(z) & =\int_{C} \log \left|\lambda-z^{m}\right| d \mu_{T}(z) \\
& =\int_{C} \sum_{k=1}^{m} \log \left|\theta_{k} \lambda^{\frac{1}{m}}-z\right| d \mu_{T}(z) \\
& =\sum_{k=1}^{m} L\left(T-\theta_{k} \lambda^{\frac{1}{m}} \mathbf{1}\right) \\
& =L\left(\prod_{k=1}^{m}\left(T-\theta_{k} \lambda^{\frac{1}{m}} \mathbf{1}\right)\right) \\
& =L\left(T^{m}-\lambda \mathbf{1}\right),
\end{aligned}
$$

as desired.

Proposition 2.16. If $T \in \mathcal{M}^{\Delta}$ with

$$
\int_{0}^{1} \log t d \mu_{|T|}(t)>-\infty
$$

then $\mu_{T}(\{0\})=\mu_{|T|}(\{0\})=0$, and $T$ has an inverse $T^{-1} \in \mathcal{M}^{\Delta}$. Moreover, $\mu_{T^{-1}}$ is the push-forward measure of $\mu_{T}$ via the map $z \mapsto z^{-1}$.

Proof. According to Theorem 2.7,

$$
\int_{\mathrm{C}} \log |z| d \mu_{T}(z)=L(T)=\int_{0}^{\infty} \log t d \mu_{|T|}(t) .
$$

Hence, if (2.44) holds, then

$$
-\infty<\int_{C} \log |z| d \mu_{T}(z)<\infty,
$$

and therefore $\mu_{T}(\{0\})=\mu_{|T|}(\{0\})=0$. Moreover, $|T|$ has an inverse $|T|^{-1} \in$ $\tilde{M}$ with

$$
\begin{aligned}
\int_{0}^{\infty} \log ^{+}(t) d \mu_{|T|^{-1}}(t) & =\int_{0}^{\infty} \log ^{+}\left(\frac{1}{t}\right) d \mu_{|T|}(t) \\
& =-\int_{0}^{1} \log t d \mu_{|T|}(t)<\infty
\end{aligned}
$$

so $|T|^{-1} \in \mathscr{M}^{\Delta}$. Take $U \in \mathscr{U}(\mathscr{M})$ such that $T=U|T|$. Then $T^{-1}=$ $|T|^{-1} U^{*} \in \mathscr{M}^{\Delta}$. 
Now, let $v$ denote the push-forward measure of $\mu_{T}$ under the map $z \mapsto z^{-1}$. According to Theorem 2.7, if

$$
\int_{C} \log ^{+}|z| d v(z)<\infty
$$

and

$$
\forall \lambda \in \mathrm{C}: \quad \int_{\mathrm{C}} \log |z-\lambda| d \nu(z)=L\left(T^{-1}-\lambda \mathbf{1}\right),
$$

then $v=\mu_{T^{-1}}$. Applying (2.46) we find that

$$
\int_{C} \log ^{+}|z| d \nu(z)=\int_{C} \log ^{+}\left|\frac{1}{z}\right| d \mu_{T}(z)=-\int_{(|z| \leq 1)} \log |z| d \mu_{T}(z)<\infty .
$$

In order to prove that (2.48) holds, let $\lambda \in C$. If $\lambda \neq 0$, then, using the multiplicativity of $\Delta$ on $\mathscr{M}^{\Delta}$, we find that

$$
\begin{aligned}
\int_{C} \log |z-\lambda| d v(z) & =\int_{C} \log \left|\frac{1}{z}-\lambda\right| d \mu_{T}(z) \\
& =\int_{C} \log \left|\frac{1}{z}\left(\frac{1}{\lambda}-z\right) \lambda\right| d \mu_{T}(z) \\
& =\int_{C}\left(\log |\lambda|+\log \left|\frac{1}{\lambda}-z\right|-\log |z|\right) d \mu_{T}(z) \\
& =L(\lambda \mathbf{1})+L\left(T-\frac{1}{\lambda} \mathbf{1}\right)-L(T) \\
& =L\left(\lambda \mathbf{1}\left(T-\frac{1}{\lambda} \mathbf{1}\right) T^{-1}\right) \\
& =L\left(T^{-1}-\lambda \mathbf{1}\right) .
\end{aligned}
$$

In the case $\lambda=0$ we have:

$$
\int_{\mathrm{C}} \log |z| d v(z)=-\int_{\mathrm{C}} \log |z| d \mu_{T}(z)=-L(T)=L\left(T^{-1}\right) .
$$

Hence (2.48) holds, and $v=\mu_{T^{-1}}$.

Proposition 2.17. Let $T \in \mathcal{M}^{\Delta}$. Then $\operatorname{supp}\left(\mu_{T}\right) \subseteq \sigma(T)$.

Proof. Let $\lambda \in \mathrm{C} \backslash \sigma(T)$. Then $T-\lambda \mathbf{1}$ is invertible with bounded inverse. Moreover, according to Proposition 2.16, $\mu_{(T-\lambda \mathbf{1})^{-1}}$ is the push-forward measure of $\mu_{T-\lambda \mathbf{1}}$ via the map $z \mapsto z^{-1}, z \in C \backslash\{0\}$. Since $(T-\lambda \mathbf{1})^{-1}$ is bounded, 
we have from [3] that

$$
\operatorname{supp}\left(\mu_{(T-\lambda \mathbf{1})^{-1}} \subseteq \sigma\left((T-\lambda \mathbf{1})^{-1}\right) \subseteq \overline{B(0, r)}\right.
$$

where $r=\left\|(T-\lambda \mathbf{1})^{-1}\right\|$. Hence,

$$
\operatorname{supp}\left(\mu_{T-\lambda \mathbf{1}}\right) \subseteq\left\{z \in \mathrm{C}|| z \mid \geq \frac{1}{r}\right\} .
$$

In particular, $0 \notin \operatorname{supp}\left(\mu_{T-\lambda \mathbf{1}}\right)$, which by Proposition 2.14 is equivalent to $\lambda \notin \operatorname{supp}\left(\mu_{T}\right)$. Hence, $\operatorname{supp}\left(\mu_{T}\right) \subseteq \sigma(T)$.

Lemma 2.18. For every $p \in(0, \infty)$ and every $t \in[0, \infty[$,

$$
t^{p}=p^{2} \int_{0}^{\infty} \log ^{+}(a t) a^{-p-1} d a .
$$

Proof. For $t=0$ this is trivial. For $t>0$ we find that

$$
\begin{aligned}
\int_{0}^{\infty} \log ^{+}(a t) a^{-p-1} d a & =\int_{\frac{1}{t}}^{\infty} \log (a t) a^{-p-1} d a \\
& =\left[-\frac{1}{p} \log (a t) a^{-p}\right]_{\frac{1}{t}}^{\infty}-\int_{\frac{1}{t}}^{\infty}-\frac{1}{p a} a^{-p} d a \\
& =0-\left[-\frac{1}{p^{2}} a^{-p}\right]_{\frac{1}{t}}^{\infty} \\
& =\frac{1}{p^{2}} t^{p} .
\end{aligned}
$$

We will now prove Weil's inequality for operators $T$ in $L^{p}(\mathcal{M})$ (cf. [3, corollary 3.8] for the case $T \in \mathcal{M}$ ):

Theorem 2.19. Let $p \in(0, \infty)$ and let $T \in L^{p}(\mathcal{M})$. Then

$$
\int_{C}|z|^{p} d \mu_{T}(z) \leq\|T\|_{p}^{p} .
$$

In the proof of this theorem we shall need the following lemma, the proof of which we postpone for a while:

Lemma 2.20. Let $T \in \mathcal{M}^{\Delta}$. Then

$$
\int_{C} \log ^{+}|z| d \mu_{T}(z) \leq \tau\left(\log ^{+}|T|\right) .
$$


Proof of Theorem 2.19. Let $a \geq 0$. Then, according to Lemma 2.14 and Lemma 2.20,

$$
\begin{aligned}
\int_{\mathrm{C}} \log ^{+}(a|z|) d \mu_{T}(z) & =\int_{\mathrm{C}} \log ^{+}|z| d \mu_{a T}(z) \\
& \leq \int_{0}^{\infty} \log ^{+} t d \mu_{|a T|}(t) \\
& =\int_{0}^{\infty} \log ^{+}(a t) d \mu_{|T|}(t) .
\end{aligned}
$$

Hence by Lemma 2.18 and Tonelli's Theorem,

$$
\begin{aligned}
\int_{\mathrm{C}}|z|^{p} d \mu_{T}(z) & =p^{2} \int_{0}^{\infty}\left(\int_{\mathrm{C}} \log ^{+}(a|z|) d \mu_{T}(z)\right) a^{-p-1} d a \\
& \leq p^{2} \int_{0}^{\infty}\left(\int_{0}^{\infty} \log ^{+}(a t) d \mu_{|T|}(t)\right) a^{-p-1} d a \\
& =\int_{0}^{\infty} t^{p} d \mu_{|T|}(t)=\tau\left(|T|^{p}\right) .
\end{aligned}
$$

In order to prove Lemma 2.20 we shall need some additional results:

Lemma 2.21. Suppose $A, B, C \in \mathscr{M}^{\Delta}$ with $A$ and $B$ invertible in $\mathscr{M}^{\Delta}$ and

$$
\left(\begin{array}{cc}
A & C^{*} \\
C & B
\end{array}\right) \geq 0
$$

Then

$$
\Delta(C) \leq \Delta(A)^{\frac{1}{2}} \Delta(B)^{\frac{1}{2}} .
$$

Proof. Note that $A, B \geq 0$ and that

$$
\begin{aligned}
\left(\begin{array}{cc}
\mathbf{1} & A^{-\frac{1}{2}} C^{*} B^{-\frac{1}{2}} \\
B^{-\frac{1}{2}} C A^{-\frac{1}{2}} & \mathbf{1}
\end{array}\right) & =\left(\begin{array}{cc}
A^{-\frac{1}{2}} & 0 \\
0 & B^{-\frac{1}{2}}
\end{array}\right)\left(\begin{array}{cc}
A & C^{*} \\
C & B
\end{array}\right)\left(\begin{array}{cc}
A^{-\frac{1}{2}} & 0 \\
0 & B^{-\frac{1}{2}}
\end{array}\right) \\
& \geq 0,
\end{aligned}
$$

which is equivalent to saying that $\left\|B^{-\frac{1}{2}} C A^{-\frac{1}{2}}\right\| \leq 1$, and this clearly implies that

$$
\Delta\left(B^{-\frac{1}{2}} C A^{-\frac{1}{2}}\right) \leq 1 \text {. }
$$


Lemma 2.22. For every $S \in \mathscr{M}^{\Delta}$,

$$
\Delta(\mathbf{1}+S) \leq \Delta(\mathbf{1}+|S|) .
$$

Proof. Take a unitary $U \in \mathscr{M}$ such that $S=U|S|$. Then

$$
\left(\begin{array}{ll}
|S| & |S| \\
|S| & |S|
\end{array}\right) \geq 0
$$

and

$$
\left(\begin{array}{cc}
\mathbf{1} & U^{*} \\
U & \mathbf{1}
\end{array}\right) \geq 0
$$

whence

$$
\left(\begin{array}{cc}
|S|+\mathbf{1} & |S|+U^{*} \\
|S|+U & |S|+\mathbf{1}
\end{array}\right) \geq 0
$$

Now Lemma 2.21 implies that

$$
\begin{aligned}
\Delta(S+\mathbf{1}) & =\Delta\left(U^{*}(S+\mathbf{1})\right)=\Delta\left(U^{*}(U|S|+\mathbf{1})\right) \\
& =\Delta\left(|S|+U^{*}\right) \leq \Delta(|S|+\mathbf{1})^{\frac{1}{2}} \Delta(|S|+\mathbf{1})^{\frac{1}{2}}=\Delta(|S|+\mathbf{1}),
\end{aligned}
$$

as desired.

Lemma 2.23. Every $S \in \mathscr{M}^{\Delta}$ satisfies

$$
\Delta\left(\mathbf{1}+\left|S^{2}\right|\right) \leq \Delta\left(\mathbf{1}+|S|^{2}\right)
$$

implying that for arbitrary $n \in \mathrm{N}$,

$$
\Delta\left(\mathbf{1}+\left|S^{2^{n}}\right|\right) \leq \Delta\left(\mathbf{1}+|S|^{2^{n}}\right) .
$$

Proof. Take a unitary $U \in \mathscr{M}$ such that $S^{2}=U\left|S^{2}\right|$. Since

$$
\left(\begin{array}{cc}
S S^{*} & S^{2} \\
\left(S^{*}\right)^{2} & S^{*} S
\end{array}\right)=\left(\begin{array}{c}
S \\
S^{*}
\end{array}\right)\left(\begin{array}{cc}
S^{*} & S
\end{array}\right) \geq 0
$$

we find as in the foregoing proof that

$$
\left(\begin{array}{cc}
\mathbf{1}+S S^{*} & U^{*}+S^{2} \\
U+\left(S^{*}\right)^{2} & \mathbf{1}+S^{*} S
\end{array}\right) \geq 0
$$

Again this implies that

$$
\Delta\left(\mathbf{1}+\left|S^{2}\right|\right)=\Delta\left(S^{2}+U^{*}\right) \leq \Delta\left(\mathbf{1}+S^{*} S\right)^{\frac{1}{2}} \Delta\left(\mathbf{1}+S S^{*}\right)^{\frac{1}{2}}=\Delta\left(\mathbf{1}+S^{*} S\right),
$$


where the last identity follows from the fact that $S^{*} S$ and $S S^{*}$ have the same distribution w.r.t. $\tau$.

Proof of Lemma 2.20. According to (2.38) we have:

$$
\int_{\mathrm{C}} \log ^{+}|z| d \mu_{T}(z)=\frac{1}{2 \pi} \int_{0}^{2 \pi} f\left(e^{i \theta}\right) d \theta,
$$

where

$$
f(\lambda)=\tau(\log |T-\lambda \mathbf{1}|)=\log \Delta(T-\lambda \mathbf{1}), \quad \lambda \in \mathrm{C} .
$$

For every positive integer $n$ define $f_{n}$ by

$$
f_{n}(z)=\sum_{k=0}^{2^{n}-1} f\left(e^{\frac{2 \pi k}{2^{n}} i} z\right), \quad z \in \mathrm{C} .
$$

Then clearly,

$$
\frac{1}{2 \pi} \int_{0}^{2 \pi} f\left(e^{i \theta}\right) d \theta=\frac{1}{2 \pi 2^{n}} \int_{0}^{2 \pi} f_{n}\left(e^{i \theta}\right) d \theta .
$$

Applying Lemma 2.22 and Lemma 2.23 we obtain an estimate of $f_{n}\left(e^{i \theta}\right)$ :

$$
\begin{aligned}
f_{n}\left(e^{i \theta}\right) & =\sum_{k=0}^{2^{n}-1} \log \Delta\left(e^{-i \theta} e^{-\frac{2 \pi k}{2^{n}} i} T-\mathbf{1}\right)=\log \Delta\left(\prod_{k=0}^{2^{n}-1}\left(e^{-i \theta} e^{-\frac{2 \pi k}{2^{n}} i} T-\mathbf{1}\right)\right) \\
& =\log \Delta\left(\mathbf{1}-e^{-i 2^{n} \theta} T^{2^{n}}\right) \leq \log \Delta\left(\mathbf{1}+\left|T^{2^{n}}\right|\right) \leq \log \Delta\left(\mathbf{1}+|T|^{2^{n}}\right) \\
& =\tau\left(\log \left(\mathbf{1}+|T|^{2^{n}}\right)\right) .
\end{aligned}
$$

Combining (2.56) and (2.59) with the above estimate we see that

$$
\begin{aligned}
\int_{\mathrm{C}} \log ^{+}|z| d \mu_{T}(z) \leq & \frac{1}{2^{n}} \tau\left(\log \left(\mathbf{1}+|T|^{2^{n}}\right)\right) \\
= & \frac{1}{2^{n}} \int_{[0, \infty[} \log \left(1+t^{2^{n}}\right) d \mu_{|T|}(t) \\
\leq & \frac{1}{2^{n}} \int_{[0,1[} \log 2 d \mu_{|T|}(t) \\
& +\frac{1}{2^{n}} \int_{[1, \infty[}\left(\log 2+2^{n} \log t\right) d \mu_{|T|}(t) \\
\leq & \frac{2 \log 2}{2^{n}}+\int_{[0, \infty[} \log ^{+} t d \mu_{|T|}(t) .
\end{aligned}
$$


Finally, let $n \rightarrow \infty$, and conclude that

$$
\int_{\mathrm{C}} \log ^{+}|z| d \mu_{T}(z) \leq \int_{[0, \infty[} \log ^{+} t d \mu_{|T|}(t) .
$$

Proposition 2.24. Let $T \in \mathcal{M}^{\Delta}$, and suppose $P \in \mathcal{M}$ is a non-trivial $T$-invariant projection, i.e. $P T P=T P$. Then

$$
\Delta(T)=\Delta_{P \mathscr{M} P}(P T P)^{\tau(P)} \Delta_{P \perp \mathscr{M} P^{\perp}}\left(P^{\perp} T P^{\perp}\right)^{1-\tau(P)},
$$

where $\Delta_{P M P}$ and $\Delta_{P^{\perp} M P^{\perp}}$ refer to the Fuglede-Kadison determinant computed relative to the normalized traces $\left.\frac{1}{\tau(P)} \tau\right|_{P \mathscr{M} P}$ and $\left.\frac{1}{\tau\left(P^{\perp}\right)} \tau\right|_{P^{\perp} M_{P^{\perp}} \text { on } P \mathscr{M} P}$ and $P^{\perp} M P^{\perp}$, respectively.

Proof. Put $T_{11}=P T P, T_{12}=P T P^{\perp}$ and $T_{22}=P^{\perp} T P^{\perp}$. Then, w.r.t. to the decomposition $\mathscr{H}=P(\mathscr{H}) \oplus P(\mathscr{H})^{\perp}$, we may write

$$
T=\left(\begin{array}{cc}
T_{11} & T_{12} \\
0 & T_{22}
\end{array}\right)=\left(\begin{array}{cc}
\mathbf{1} & 0 \\
0 & T_{22}
\end{array}\right)\left(\begin{array}{cc}
\mathbf{1} & T_{12} \\
0 & \mathbf{1}
\end{array}\right)\left(\begin{array}{cc}
T_{11} & 0 \\
0 & \mathbf{1}
\end{array}\right),
$$

where

$$
\Delta\left(\left(\begin{array}{cc}
\mathbf{1} & 0 \\
0 & T_{22}
\end{array}\right)\right)=\Delta_{P^{\perp} M P^{\perp}}\left(P^{\perp} T P^{\perp}\right)^{1-\tau(P)},
$$

and

$$
\Delta\left(\left(\begin{array}{cc}
T_{11} & 0 \\
0 & \mathbf{1}
\end{array}\right)\right)=\Delta_{P \mathscr{M} P}(P T P)^{\tau(P)} .
$$

Thus, (2.60) holds if

$$
\Delta\left(\left(\begin{array}{cc}
\mathbf{1} & T_{12} \\
0 & \mathbf{1}
\end{array}\right)\right)=1
$$

To that (2.60) holds, note that

$$
\left(\begin{array}{cc}
\mathbf{1} & T_{12} \\
0 & \mathbf{1}
\end{array}\right)^{-1}=\left(\begin{array}{cc}
\mathbf{1} & -T_{12} \\
0 & \mathbf{1}
\end{array}\right)
$$

and hence

$$
\Delta\left(\left(\begin{array}{cc}
\mathbf{1} & T_{12} \\
0 & \mathbf{1}
\end{array}\right)\right) \Delta\left(\left(\begin{array}{cc}
\mathbf{1} & -T_{12} \\
0 & \mathbf{1}
\end{array}\right)\right)=1 .
$$

Also,

$$
\left(\begin{array}{cc}
\mathbf{1} & -T_{12} \\
0 & \mathbf{1}
\end{array}\right)=\left(\begin{array}{cc}
\mathbf{1} & 0 \\
0 & -\mathbf{1}
\end{array}\right)\left(\begin{array}{cc}
\mathbf{1} & T_{12} \\
0 & \mathbf{1}
\end{array}\right)\left(\begin{array}{cc}
\mathbf{1} & 0 \\
0 & -\mathbf{1}
\end{array}\right),
$$


so that

$$
\Delta\left(\left(\begin{array}{cc}
\mathbf{1} & -T_{12} \\
0 & \mathbf{1}
\end{array}\right)\right)=\Delta\left(\left(\begin{array}{cc}
\mathbf{1} & T_{12} \\
0 & \mathbf{1}
\end{array}\right)\right),
$$

and then by (2.62),

$$
\Delta\left(\left(\begin{array}{cc}
\mathbf{1} & T_{12} \\
0 & \mathbf{1}
\end{array}\right)\right)=1,
$$

as desired.

Lemma 2.25. Let $p \in(0, \infty)$, and let $\varepsilon>0$. Then the map $L_{\varepsilon}: L^{p}(\mathcal{M}, \tau)$ $\rightarrow \mathrm{R}$ given by

$$
L_{\varepsilon}(T)=\frac{1}{2} \tau\left(\log \left(T^{*} T+\varepsilon \mathbf{1}\right)\right), \quad T \in L^{p}(\mathscr{M}, \tau),
$$

is continuous w.r.t. $\|\cdot\|_{p}$.

Proof. Suppose $T, T_{n} \in L^{p}(\mathscr{M}, \tau)$ with

$$
\lim _{n \rightarrow \infty}\left\|T-T_{n}\right\|_{p}=0 .
$$

Then $T_{n} \rightarrow T$ in the measure topology (cf. [6]). Therefore, $T_{n}^{*} T_{n} \rightarrow T^{*} T$ in measure, and then with respect to the weak topology on $\operatorname{Prob}(R)$,

$$
\mu_{T^{*} T}=\lim _{n \rightarrow \infty} \mu_{T_{n}^{*} T_{n}} .
$$

Define a sequence $\left(v_{n}\right)_{n=1}^{\infty}$ of (finite) measures on $(\mathrm{R}, \mathrm{B})$ by

$$
d v_{n}(t)=\left(1+t^{\frac{p}{2}}\right) d \mu_{T_{n}^{*} T_{n}}(t),
$$

and note that since $\lim _{n \rightarrow \infty}\left\|T_{n}\right\|_{p}=\|T\|_{p}$,

$$
\sup _{n \in \mathrm{N}} v_{n}(\mathrm{R})<\infty .
$$

Similarly, define a finite measure $v$ on $(\mathrm{R}, \mathrm{B})$ by

$$
d v(t)=\left(1+t^{\frac{p}{2}}\right) d \mu_{T^{*} T}(t) .
$$

Because of (2.64), we have that for every $\phi \in C_{c}(\mathrm{R})$,

$$
\int_{0}^{\infty} \phi(t) d v(t)=\lim _{n \rightarrow \infty} \int_{0}^{\infty} \phi(t) d v_{n}(t) .
$$

When $\phi \in C_{0}(\mathrm{R}), \phi$ may be approximated (uniformly) by functions from $C_{c}(\mathrm{R})$. Thus, taking (2.66) and (2.68) into account, one easily sees that

$$
\int_{0}^{\infty} \phi(t) d v(t)=\lim _{n \rightarrow \infty} \int_{0}^{\infty} \phi(t) d v_{n}(t) .
$$


In particular, with

$$
\phi(t)=\frac{\log (t+\varepsilon)}{1+t^{\frac{p}{2}}}, \quad t \geq 0
$$

(2.69) implies that

$$
L_{\varepsilon}(T)=\int_{0}^{\infty} \phi(t) d v(t)=\lim _{n \rightarrow \infty} \int_{0}^{\infty} \phi(t) d v_{n}(t)=\lim _{n \rightarrow \infty} L_{\varepsilon}\left(T_{n}\right) .
$$

Corollary 2.26. For $p \in(0, \infty)$ the map $L: L^{p}(\mathcal{M}, \tau) \rightarrow[-\infty, \infty[$ given by

$$
L(T)=\log \Delta(T), \quad T \in L^{p}(\mathcal{M}, \tau),
$$

is upper semicontinuous w.r.t. $\|\cdot\|_{p}$.

Proof. Indeed, this follows from Lemma 2.25, since for every $T \in$ $L^{p}(\mathscr{M}, \tau)$ we have that

$$
L(T)=\inf _{\varepsilon>0} L_{\varepsilon}(T)
$$

\section{Unbounded $R$-diagonal operators}

Consider a von Neumann algebra $\mathscr{M}$ equipped with a faithful, normal, tracial state $\tau$.

Definition 3.1. For $T \in \tilde{\mathscr{M}}$ with polar decomposition $T=U|T|$, we denote by $W^{*}(T)$ the von Neumann algebra generated by $U$ and all the spectral projections of $|T|$.

Note that $T$ is affiliated with $W^{*}(T)$ and that $W^{*}(T)$ is the smallest von Neumann subalgebra of $\mathscr{M}$ with this property.

If $\mathscr{M}_{1}$ and $\mathscr{M}_{2}$ are finite von Neumann algebras with faithful, normal, tracial states $\tau_{1}$ and $\tau_{2}$, respectively, then any $*$-isomorphism $\phi: \mathscr{M}_{1} \rightarrow \mathscr{M}_{2}$ with $\tau_{1}=\tau_{2} \circ \phi$ is continuous w.r.t. the measure topologies on the two von Neumann algebras and thus has a unique extension to a (surjective) $*$-isomorphism $\tilde{\phi}$ : $\tilde{\mathscr{M}}_{1} \rightarrow \tilde{\mathscr{M}}_{2}$.

Definition 3.2. Let $S, T \in \tilde{M}$.

(a) We say that $S$ and $T$ have the same *-distribution, in symbols $S \underset{* \mathscr{D}}{\sim} T$, if there exists a trace-preserving $*$-isomporphism $\phi$ from $W^{*}(S)$ onto $W^{*}(T)$ with $\tilde{\phi}(S)=T$.

(b) We say that $S$ and $T$ are $*$-free if $W^{*}(S)$ and $W^{*}(T)$ are $*$-free. 
Note that in case $S$ and $T$ are bounded, the two definitions (a) and (b) given above agree with the ones given in [18] .

Recall from [16, p. 155ff.] that if $U, H \in \mathcal{M}$ are $*$-free elements with $U$ Haar unitary, then $U H$ is $R$-diagonal in the sense of Nica and Speicher (cf. [16]). Conversely, if $T \in \mathcal{M}$ is $R$-diagonal, then $T$ has the same $*$-distribution as a product $U H$, where $U$ and $H$ are $*$-free elements in some tracial $C^{*}$ probability space, $U$ is a Haar unitary, and $H \geq 0$. We therefore define $R$ diagonality for operators in $\tilde{\mathscr{M}}$ as follows:

Definition 3.3. $T \in \tilde{\mathscr{M}}$ is said to be $R$-diagonal if there exist a von Neumann algebra $\mathcal{N}$, with a faithful, normal, tracial state, and $*$-free elements $U$ and $H$ in $\tilde{\mathcal{N}}$, such that $U$ is Haar unitary, $H \geq 0$, and such that $T$ has the same $*$-distribution as $U H$.

Remark 3.4. Note that if $T \in \tilde{M}$ is $R$-diagonal with $\operatorname{ker}(T)=0$, then the partial isometry $V$ in the polar decomposition of $T, T=V|T|$, is a unitary ( $\mathcal{M}$ is finite). It follows from Definition 3.3 and Definition 3.2 that $V$ is in fact a Haar unitary which is $*$-free from $|T|$.

In this section we will see that certain algebraic operations on (sets of *free) $R$-diagonal operators preserve $R$-diagonality, exactly as in the bounded case (cf. [9]). Our proofs are to a large extent inspired by the techniques used in [9] and in [14]. In particular, we will repeatedly make use of [9, Lemma 3.7] which we state here for the convenience of the reader:

Lemma 3.5. [9] Let $U \in \mathcal{M}$ be a Haar unitary, and suppose $\mathscr{S} \subset \mathcal{M}$ is a set which is $*$-free from $U$. Then for any $n \in \mathrm{N}$,

(i) the sets $\mathscr{S}, U \mathscr{S} U^{*}, U^{2} \mathscr{S}\left(U^{*}\right)^{2}, \ldots$ are $*$-free,

(ii) the sets $\mathscr{S}, U \mathscr{S} U^{*}, \ldots, U^{n-1} \mathscr{S}\left(U^{*}\right)^{n-1},\left\{U^{n}\right\}$ are $*$-free,

(iii) the sets $U \mathscr{S} U^{*}, \ldots, U^{n} \mathscr{S}\left(U^{*}\right)^{n},\left\{U^{n}\right\}$ are $*$-free.

Proposition 3.6. If $T \in \tilde{M}$ is $R$-diagonal with $\operatorname{ker}(T)=0$, then $T$ has an inverse $T^{-1} \in \tilde{M}$, and $T^{-1}$ is $R$-diagonal as well.

Proof. Let $T=V|T|$ be the polar decomposition of $T$ with $V \in \mathscr{M}$ Haar unitary and $*$-free from $|T|$. Since $\operatorname{ker}(T)=0, T$ has an inverse $T^{-1} \in \tilde{\mathscr{M}}$ :

$$
T^{-1}=V^{*} V|T|^{-1} V^{*}=V^{*}\left(V|T| V^{*}\right)^{-1},
$$

where $V^{*}$ is Haar unitary and, according to Lemma 3.5 , it is $*$-free from $V|T| V^{*}$ and thus from $\left(V|T| V^{*}\right)^{-1}$. This shows that $T^{-1}$ is $R$-diagonal.

Lemma 3.7. Let $S, T \in \tilde{M}$, and let $V \in \mathcal{M}$ be a Haar unitary. If $S, T$ and $V$ are $*$-free, then $V S$ and $T V S$ are $R$-diagonal. 
Proof. The case where $S$ and $T$ are bounded was treated by F. Larsen (cf. [14, Lemma 3.6]). Our proof resembles the one given by F. Larsen.

Enlarging the algebra if necessary, we may assume that there are Haar unitaries $V_{1}, V_{2} \in \mathcal{M}$, such that $V_{1}, V_{2}$ and $S$ are $*$-free and $V=V_{1} V_{2}$.

Since $W^{*}(S) \subseteq \mathscr{M}$ is finite, there is a unitary $U_{1} \in W^{*}(S)$ such that $S=U_{1}|S|$. Then $V S=V_{1}\left(V_{2} U_{1}\right)|S|$, where

(i) $V_{1}$ is $*$-free from $|S|$ and $V_{2} U_{1}$,

(ii) $\tau\left(V_{1}\right)=\tau\left(V_{1}^{*}\right)=0$ and $\tau\left(V_{2} U_{1}\right)=\tau\left(\left(V_{2} U_{1}\right)^{*}\right)=0$,

(ii) for all $A \in W^{*}(|S|)$ with $\tau(A)=0, \tau\left(V_{2} U_{1} A\right)=\tau\left(V_{2}\right) \tau\left(U_{1} A\right)=0$, $\tau\left(A U_{1}^{*} V_{2}^{*}\right)=\tau\left(A U_{1}^{*}\right) \tau\left(V_{2}^{*}\right)=0$ and $\tau\left(V_{2} U_{1} A\left(V_{2} U_{1}\right)^{-1}\right)=\tau(A)=$ 0 .

It follows now from [17, Lemma 2.4] that $V_{1}\left(V_{2} U_{1}\right)$ is $*$-free from $|S|$. Thus, if $V_{1}\left(V_{2} U_{1}\right)$ is Haar unitary, then $V S$ is $R$-diagonal. By (ii) and the freeness of $V_{1}$ and $V_{2} U$, we have that for all $n \in \mathrm{N}$,

$$
\tau\left(\left(V_{1} V_{2} U_{1}\right)^{n}\right)=\tau\left(V_{1}\left(V_{2} U_{1}\right) V_{1}\left(V_{2} U_{1}\right) \cdots V_{1}\left(V_{2} U_{1}\right)\right)=0 .
$$

Then $\tau\left(\left(V_{1} V_{2} U_{1}\right)^{-n}\right)=\overline{\tau\left(\left(V_{1} V_{2} U_{1}\right)^{n}\right)}=0, n \in \mathrm{N}$. That is, $V_{1} V_{2} U_{1}$ is Haar unitary, and it follows that $V S=V_{1} V_{2} U_{1}|S|$ is $R$-diagonal.

Now, $T V S=V\left(V^{*} T V S\right)$. Put

$$
\mathscr{B}_{1}=W^{*}(V), \quad \mathscr{B}_{2}=W^{*}(T), \quad \text { and } \quad \mathscr{B}_{3}=W^{*}(S) .
$$

Then $\mathscr{B}_{1}, \mathscr{B}_{2}$ and $\mathscr{B}_{3}$ are $*$-free. We may write $T$ as $T=U_{2}|T|$ for a unitary $U_{2} \in \mathscr{B}_{2}$. Then

$$
V^{*} T V=\left(V^{*} U_{2} V\right) V^{*}|T| V
$$

where $V^{*}|T| V$ is affiliated with $V^{*} \mathscr{B}_{2} V$.

$\mathscr{B}_{3}$ and $V^{*} \mathscr{B}_{2} V$ are $*$-free, and according to Lemma $3.5, \mathscr{B}_{1}$ and $V^{*} \mathscr{B}_{2} V$ are $*$-free. But then $V$ is $*$-free from $\mathscr{B}_{4}=\mathscr{B}_{3} \vee V^{*} \mathscr{B}_{2} V$.

Since $S$ and $V^{*} T V$ are both affiliated with $\mathscr{B}_{4}$, their product, $V^{*} T V S$, is affiliated with $\mathscr{B}_{4}$, so $V$ is $*$-free from $V^{*} T V S$. It follows now from the first part of the proof that $T V S=V\left(V^{*} T V S\right)$ is $R$-diagonal.

Proposition 3.8. If $S, T \in \tilde{M}$ are $*$-free $R$-diagonal elements, then $S T$ is $R$-diagonal as well. Moreover,

$$
\mu_{(S T) * S T}=\mu_{S * S} \otimes \mu_{T * T} .
$$

Proof. Taking a free product of tracial von Neumann algebras if necessary, we can find a von Neumann algebra $\mathcal{N}$ with faithful, normal, tracial state $\omega$ 
and $*$-free elements $U_{1}, H_{1}, U_{2}, H_{2} \in \tilde{\mathcal{N}}$ such that $U_{1}, U_{2}$ are Haar unitaries, $H_{1}, H_{2} \geq 0$, and $S \underset{* \mathscr{D}}{\sim} U_{1} H_{1}$ and $T \underset{* \mathscr{D}}{\sim} U_{2} H_{2}$.

Choose trace-preserving $*$-isomorphisms

$$
\begin{aligned}
\phi_{1}: W^{*}(S) & \rightarrow W^{*}\left(U_{1} H_{1}\right), \\
\phi_{2}: W^{*}(T) & \rightarrow W^{*}\left(U_{2} H_{2}\right),
\end{aligned}
$$

with $\tilde{\phi}_{1}(S)=U_{1} H_{1}$ and $\tilde{\phi}_{2}(T)=U_{2} H_{2} . \phi_{1}$ and $\phi_{2}$ give rise to a tracepreserving $*$-isomorphism

$$
\phi=\phi_{1} * \phi_{2}: W^{*}(S) * W^{*}(T) \rightarrow W^{*}\left(U_{1} H_{1}\right) * W^{*}\left(U_{2} H_{2}\right)
$$

(the free products are taken within the category of tracial von Neumann algebras) with

$$
\tilde{\phi}(S T)=\tilde{\phi}_{1}(S) \tilde{\phi}_{2}(T)=U_{1} H_{1} U_{2} H_{2} .
$$

Thus, $\psi:=\left.\phi\right|_{W^{*}(S T)}$ is a trace-preserving $*$-isomorphism onto $W^{*}\left(U_{1} H_{1} U_{2} H_{2}\right)$ with $\tilde{\psi}(S T)=U_{1} H_{1} U_{2} H_{2}$. According to Lemma 3.7, $U_{1}\left(H_{1} U_{2} H_{2}\right)$ is $R$ diagonal, and hence $S T$ is $R$-diagonal.

In order to prove (3.2), note that if $S=0$, then $\mu_{S^{*} S}=\delta_{0}$, so that by the definition of multiplicative free convolution given on p. 744 in [4],

$$
\mu_{S^{*} S} \otimes \mu_{T^{*} T}=\delta_{0} \otimes \mu_{T^{*} T}=\delta_{0} .
$$

This shows that $\mu_{S^{*} S} \otimes \mu_{T^{*} T}=\mu_{(S T)^{*} S T}$ if $S=0$. The same holds if $T=0$.

Now assume that $S, T \neq 0$. Note that

$$
\begin{aligned}
& S^{*} S \underset{* \mathscr{D}}{\sim} H_{1}^{2}, \\
& T^{*} T \underset{* \mathscr{D}}{\sim} H_{2}^{2}, \\
&(S T)^{*} S T \underset{* \mathscr{D}}{\sim} H_{2} U_{2}^{*} H_{1}^{2} U_{2} H_{2} .
\end{aligned}
$$

Thus, (3.2) holds if

$$
\mu_{H_{2} U_{2}^{*} H_{1}^{2} U_{2} H_{2}}=\mu_{H_{1}^{2}} \otimes \mu_{H_{2}^{2}} .
$$

For every $n \in \mathrm{N}$, the bounded operators

$$
S_{n}=U_{1} H_{1} 1_{[0, n]}\left(H_{1}\right) \quad \text { and } \quad T_{n}=U_{2} H_{2} 1_{[0, n]}\left(H_{2}\right)
$$

are $*$-free. According to [9, Lemma 3.9] they are both $R$-diagonal in the sense of Nica and Speicher (cf. [16]). Then, by [9, Proposition 3.6],

$$
\mu_{\left(S_{n} T_{n}\right) * S_{n} T_{n}}=\mu_{S_{n}^{*} S_{n}} \otimes \mu_{T_{n}^{*} T_{n}}
$$


Since $S_{n} \rightarrow U_{1} H_{1}$ and $T_{n} \rightarrow U_{2} H_{2}$ in the measure topology, $\left(S_{n} T_{n}\right)^{*} S_{n} T_{n} \rightarrow$ $H_{2} U_{2}^{*} H_{1}^{2} U_{2} H_{2}$ in measure as well. These facts imply that $\mu_{S_{n}^{*} S_{n}} \stackrel{w^{*}}{\longrightarrow} \mu_{H_{1}^{2}}$, $\mu_{T_{n}^{*} T_{n}} \stackrel{w^{*}}{\longrightarrow} \mu_{H_{2}^{2}}$ and $\mu_{\left(S_{n} T_{n}\right)^{*} S_{n} T_{n}} \stackrel{w^{*}}{\longrightarrow} \mu_{H_{2} U_{2}^{*} H_{1}^{2} U_{2} H_{2}}$. Moreover, $\mu_{H_{1}^{2}} \neq \delta_{0}$ and $\mu_{H_{2}^{2}} \neq \delta_{0}$, because $S^{*} S$ and $T^{*} T$ are non-zero. Hence, by [4, Corollary 6.7] and by (3.3),

$$
\mu_{H_{2} U_{2}^{*} H_{1}^{2} U_{2} H_{2}}=w^{*}-\lim _{n \rightarrow \infty} \mu_{S_{n}^{*} S_{n}} \otimes \mu_{T_{n}^{*} T_{n}}=\mu_{H_{1}^{2}} \otimes \mu_{H_{2}^{2}} .
$$

Proposition 3.9. Let $S \in \tilde{M}$ be $R$-diagonal, and let $n \in \mathrm{N}$. Then $S^{n}$ is $R$-diagonal. Moreover,

$$
\mu_{\left(S^{n}\right) * S^{n}}=\mu_{S^{*} S}^{\otimes n} .
$$

Proof. Choose a von Neumann algebra $\mathcal{N}$ with faithful, normal, tracial state $\omega$ and with $*$-free elements $U, H \in \tilde{\mathcal{N}}$ such that $U$ is Haar unitary, $H \geq 0$, and $S \underset{* \mathscr{D}}{\sim} U H$. Then $S^{n} \underset{* \mathscr{D}}{\sim}(U H)^{n}$. Since

$$
(U H)^{n}=U^{n}\left[U^{1-n} H U^{n-1}\right]\left[U^{2-n} H U^{n-2}\right] \cdots\left[U^{-1} H U\right] H,
$$

where

$$
U^{n}, U^{1-n} H U^{n-1}, U^{2-n} H U^{n-2}, \ldots, U^{-1} H U, H
$$

are $*$-free (cf. Lemma 3.5 (ii)), and $U^{n}$ is Haar unitary, Lemma 3.7 gives us that $(U H)^{n}$ is $R$-diagonal, and hence $S^{n}$ is.

In order to prove (3.4), note that if $\mu_{S^{*} S}=\delta_{0}$, then $S=S^{n}=0$ and (3.4) trivially holds.

Now assume that $\mu_{S^{*} S} \neq \delta_{0}$. For $k \in \mathrm{N}$ define $S_{k} \in \mathcal{M}$ and $T_{k} \in \mathcal{N}$ by

$$
S_{k}=S 1_{[0, k]}(|S|) \quad \text { and } \quad T_{k}=U H 1_{[0, k]}(H) .
$$

Then $T_{k} \sim S_{* \mathscr{D}}$. Moreover, by Lemma 3.7, $T_{k}$ is $R$-diagonal in the sense of Nica and Speicher, so $S_{k}$ is $R$-diagonal. It now follows from [9, Proposition 3.10] that

$$
\mu_{\left[\left(S_{k}\right)^{n}\right]^{*}\left(S_{k}\right)^{n}}=\mu_{\left[\left(T_{k}\right)^{n}\right]^{*}\left(T_{k}\right)^{n}}=\mu_{T_{k}^{*} T_{k}}^{\bigotimes n}=\mu_{S_{k}^{*} S_{k}}^{\otimes n} .
$$

As $k$ tends to infinity, $S_{k}^{*} S_{k} \rightarrow S^{*} S$ and $\left[\left(S_{k}\right)^{n}\right]^{*}\left(S_{k}\right)^{n} \rightarrow\left(S^{n}\right)^{*} S^{n}$ in the measure topology. Since $\mu_{S^{*} S} \neq \delta_{0}$, we infer from [4, Corollary 6.7] and from (3.5) that

$$
\mu_{\left(S^{n}\right)^{*} S^{n}}=w^{*}-\lim _{k \rightarrow \infty} \mu_{\left[\left(S_{k}\right)^{n}\right]^{*}\left(S_{k}\right)^{n}}=w^{*}-\lim _{k \rightarrow \infty} \mu_{S_{k}^{*} S_{k}}^{\bigotimes n}=\mu_{S^{*} S}^{\bigotimes n} .
$$


Definition 3.10. For $\mu \in \operatorname{Prob}(\mathrm{R}, \mathrm{B})$ let $\tilde{\mu}$ denote the symmetrization of $\mu$. That is, $\tilde{\mu} \in \operatorname{Prob}(\mathrm{R}, \mathrm{B})$ is given by

$$
\tilde{\mu}(B)=\frac{1}{2}(\mu(B)+\mu(-B)), \quad(B \in \mathrm{B}) .
$$

Proposition 3.11. Let $S, T \in \tilde{M}$ be $*$-free R-diagonal elements. Then

$$
\tilde{\mu}_{|S+T|}=\tilde{\mu}_{|S|} \boxplus \tilde{\mu}_{|T|} .
$$

Proof. As in the proof of Proposition 3.8, choose $(\mathcal{N}, \omega)$ and $*$-free elements $U_{1}, H_{1}, U_{2}, H_{2} \in \tilde{\mathcal{N}}$ such that $U_{1}, U_{2}$ are Haar unitaries, $H_{1}, H_{2} \geq 0$, and $S \underset{* \mathscr{D}}{\sim} U_{1} H_{1}$ and $T \underset{* \mathscr{D}}{\sim} U_{2} H_{2}$.

Again, for $n \in \mathrm{N}$, let

$$
S_{n}=U_{1} H_{1} 1_{[0, n]}\left(H_{1}\right)
$$

and

$$
T_{n}=U_{2} H_{2} 1_{[0, n]}\left(H_{2}\right) .
$$

Then $S_{n}$ and $T_{n}$ are $*$-free and $R$-diagonal and therefore, according to [9, Proposition 3.5],

$$
\tilde{\mu}_{\left|S_{n}+T_{n}\right|}=\tilde{\mu}_{\left|S_{n}\right|} \boxplus \tilde{\mu}_{\left|T_{n}\right|}
$$

$\left|S_{n}\right| \rightarrow H_{1}$ and $\left|T_{n}\right| \rightarrow H_{2}$ in measure, implying that $\mu_{\left|S_{n}\right|} \stackrel{w^{*}}{\longrightarrow} \mu_{H_{1}}=\mu_{|S|}$ and $\mu_{\left|T_{n}\right|} \stackrel{w^{*}}{\longrightarrow} \mu_{H_{2}}=\mu_{|T|}$. Then we also have weak convergence of the symmetrized measures:

$$
\tilde{\mu}_{\left|S_{n}\right|} \stackrel{w^{*}}{\longrightarrow} \tilde{\mu}_{|S|}
$$

and

$$
\tilde{\mu}_{\left|T_{n}\right|} \stackrel{w^{*}}{\longrightarrow} \tilde{\mu}_{|T|} .
$$

Let $d$ denote the Lévy metric on $\operatorname{Prob}(\mathrm{R}, \mathrm{B})$ (cf. [4, p. 743]). Then $d$ induces the topology of weak convergence, and according to [4, Proposition 4.13] and the above observations,

$d\left(\tilde{\mu}_{|S|} \boxplus \tilde{\mu}_{|T|}, \tilde{\mu}_{\left|S_{n}\right|} \boxplus \tilde{\mu}_{\left|T_{n}\right|}\right) \leq d\left(\tilde{\mu}_{|S|}, \tilde{\mu}_{\left|S_{n}\right|}\right)+d\left(\tilde{\mu}_{|T|}, \tilde{\mu}_{\left|T_{n}\right|}\right) \rightarrow 0 \quad$ as $n \rightarrow \infty$.

It follows that

$$
\begin{aligned}
\tilde{\mu}_{|S|} \boxplus \tilde{\mu}_{|T|} & =w^{*}-\lim _{n \rightarrow \infty} \tilde{\mu}_{\left|S_{n}\right|} \boxplus \tilde{\mu}_{\left|T_{n}\right|} \\
& =w^{*}-\lim _{n \rightarrow \infty} \tilde{\mu}_{\left|S_{n}+T_{n}\right|} .
\end{aligned}
$$


Since $S$ and $T\left(U_{1} H_{1}\right.$ and $U_{2} H_{2}$, resp.) are $*$-free with $S \underset{* \mathscr{D}}{\sim} U_{1} H_{1}$ and $T \sim \widetilde{D}$ $U_{2} H_{2}$, it follows that $S+T \underset{* \mathscr{D}}{\sim} U_{1} H_{1}+U_{2} H_{2}$. Moreover, $\left|S_{n}+T_{n}\right| \stackrel{* \mathscr{D}}{\rightarrow}$ $\left|U_{1} H_{1}+U_{2} H_{2}\right| \underset{* \mathscr{D}}{\sim}|S+T|$ in measure, and thus $\tilde{\mu}_{\left|S_{n}+T_{n}\right|} \stackrel{w^{*}}{\longrightarrow} \tilde{\mu}_{|S+T|}$. Finally, this implies that

$$
\tilde{\mu}_{|S|} \boxplus \tilde{\mu}_{|T|}=\tilde{\mu}_{|S+T|} .
$$

We close this section by proving two simple results on the $S$-transform of probability measures on $(0, \infty)$ (cf. [4]).

For $\mu \in \operatorname{Prob}((0, \infty)$, B $)$ define $\psi_{\mu}: \mathrm{C} \backslash(0, \infty) \rightarrow \mathrm{C}$ by

$$
\psi_{\mu}(z)=\int_{0}^{\infty} \frac{1}{1-z t} d \mu(t)-1, \quad z \in \mathrm{C} \backslash(0, \infty) .
$$

Then $\psi_{\mu}$ is analytic and satisfies

(i) $\psi_{\mu}^{\prime}(t)>0, t \in(-\infty, 0)$,

(ii) $\psi_{\mu}(z) \rightarrow-1$ as $z \rightarrow-\infty$,

(iii) $\psi_{\mu}(z) \rightarrow 0$ as $z \rightarrow 0$.

Hence, $\psi_{\mu}$ maps a (connected) neighbourhood $\mathscr{U}_{\mu}$ of $(-\infty, 0)$ injectively onto a neighbourhood $\mathscr{V}_{\mu}$ of $(-1,0)$. Define $\chi_{\mu}, \mathscr{S}_{\mu}: \mathscr{V}_{\mu} \rightarrow \mathrm{C}$ by

$$
\begin{aligned}
& \chi_{\mu}(z)=\psi_{\mu}^{-1}(z), \quad z \in \mathscr{V}_{\mu}, \\
& \mathscr{S}_{\mu}(z)=\frac{z+1}{z} \chi_{\mu}(z), \quad z \in \mathscr{V}_{\mu} .
\end{aligned}
$$

Proposition 3.12. The map $\mu \mapsto \mathscr{S}_{\mu}$ is one-to-one on $\operatorname{Prob}((0, \infty), \mathrm{B})$.

Proof. Suppose $\mu, v \in \operatorname{Prob}((0, \infty), \mathrm{B})$ with $\mathscr{S}_{\mu}=\mathscr{S}_{\nu}$. That is, in a neighbourhood $\mathscr{V}=\mathscr{V}_{\mu} \cap \mathscr{V}_{v}$ of $(-1,0), \chi_{\mu}$ agrees with $\chi_{v}$. It follows that on $(-\infty, 0), \psi_{\mu}$ agrees with $\psi_{\nu}$, and then, by uniqueness of analytic continuation,

$$
\psi_{\mu}\left(\frac{1}{\lambda}\right)=\psi_{\nu}\left(\frac{1}{\lambda}\right), \quad \lambda \in \mathrm{C} \backslash[0, \infty[.
$$

That is, the Stieltjes-transforms $G_{\mu}$ and $G_{v}$ agree on C $\backslash[0, \infty[$. Recall that

$$
d \mu(x)=-\frac{1}{\pi} \lim _{y \rightarrow 0^{+}} G_{\mu}(x+i y) d x
$$

(weak convergence of measures), and similarly,

$$
d v(x)=-\frac{1}{\pi} \lim _{y \rightarrow 0^{+}} G_{\nu}(x+i y) d x .
$$

Thus $\mu=v$. 
Proposition 3.13. Let $\mathscr{M}$ be a $I I_{1}$-factor with tracial state $\tau$, and let $a \in \tilde{M}_{+}$with $\operatorname{ker}(a)=\{0\}$. Then for all $z$ in a neighbourhood of $(-1,0)$,

$$
\mathscr{S}_{\mu_{a^{-1}}}(z)=\frac{1}{\mathscr{S}_{\mu_{a}}(-1-z)} \text {. }
$$

Proof. Let $z \in \mathrm{C} \backslash[0, \infty[$. Then

$$
\begin{aligned}
\psi_{a^{-1}}(z) & =\int_{0}^{\infty} \frac{1}{1-z t} d \mu_{a^{-1}}(t)-1 \\
& =\int_{0}^{\infty} \frac{1}{1-\frac{z}{t}} d \mu_{a}(t)-1 \\
& =\int_{0}^{\infty} \frac{z}{t-z} d \mu_{a}(t),
\end{aligned}
$$

and hence

$$
\psi_{a^{-1}}\left(\frac{1}{z}\right)=-\int_{0}^{\infty} \frac{1}{1-z t} d \mu_{a}(t)=-\left(\psi_{a}(z)+1\right) .
$$

It follows that for all $z \in \mathrm{C} \backslash[0, \infty[$,

$$
z=\chi_{a}\left(\psi_{a}(z)\right)=\chi_{a}\left(-1-\psi_{a^{-1}}\left(\frac{1}{z}\right)\right),
$$

implying that $w=\psi_{a^{-1}}\left(\frac{1}{z}\right)$ satisfies

$$
\chi_{a^{-1}}(w)=\frac{1}{z}=\frac{1}{\chi_{a}(-1-w)},
$$

and thus

$$
\mathscr{S}_{\mu_{a^{-1}}}(w) \cdot \mathscr{S}_{\mu_{a}}(-1-w)=1 .
$$

(3.19) holds for all $w \in \psi_{a^{-1}}(\mathbf{C} \backslash[0, \infty[)$ and in particular for all $w$ in a neighbourhood of $(-1,0)$. 


\section{The Brown measure of an unbounded $R$-diagonal operator}

The Brown measure of a general bounded $R$-diagonal operator was computed in [9, Theorem 4.4]. We will generalize this result to unbounded $R$-diagonal elements in $\mathscr{M}^{\Delta}$. Our proof will take a different route than the one in [9]. This new approach will enable us to obtain an estimate of the $p$-norm of the resolvent $(T-\lambda \mathbf{1})^{-1}, 0<p<1$, for special $R$-diagonal elements $T$ (cf. Section 5).

Lemma 4.1. Let $T \in \tilde{M}$ be an R-diagonal element, and let $U \in M$ be a Haar unitary which is $*$-free from $T$. Then for every $\lambda \in \mathrm{C}$,

$$
|T-\lambda \mathbf{1}| \underset{* \mathscr{D}}{\sim}|T+| \lambda|U| .
$$

Proof. By passing to a larger algebra, we may assume that $T=V|T|$ where $V \in \mathscr{M}$ is a Haar unitary and $U, V$ and $|T|$ are $*$-free. The case $\lambda=0$ is trivial. For $\lambda \neq 0$, let $\alpha=-\frac{\lambda}{|\lambda|}$. Then $\alpha U^{*} V$ is a Haar unitary which is $*$-free from $T$. Hence,

$$
\alpha U^{*} V|T| \underset{* \mathscr{D}}{\sim} T
$$

Therefore,

$$
|T-\lambda \mathbf{1}| \underset{* \mathscr{D}}{\sim}\left|\alpha U^{*} V\right| T|-\lambda \mathbf{1}|=|T-\bar{\alpha} \lambda U|=|T+| \lambda|U| .
$$

Lemma 4.2. Let $T \in \tilde{M}$ be an R-diagonal operator, and define

$$
h(s)=s \tau\left(\left(T^{*} T+s^{2} \mathbf{1}\right)^{-1}\right), \quad s>0 .
$$

Moreover, for $\lambda \in \mathrm{C} \backslash\{0\}$, set

$$
h_{\lambda}(s)=s \tau\left(\left[(T-\lambda \mathbf{1})^{*}(T-\lambda \mathbf{1})+s^{2} \mathbf{1}\right]^{-1}\right) .
$$

Then there exists an $s_{\lambda}>0$ such that for $s>s_{\lambda}$,

$$
h(s)=h_{\lambda}\left(s+\frac{\sqrt{1-4|\lambda|^{2} h(s)^{2}}-1}{2 h(s)}\right) .
$$

Proof. By passing to a larger algebra, we may assume that there exists a Haar unitary $U \in \mathcal{M}$ which is $*$-free from $T$. Then, according to Lemma 4.1,

$$
|T-\lambda \mathbf{1}| \underset{* \mathscr{D}}{\sim}|T+| \lambda|U| .
$$


It follows now from Proposition 3.11 that

$$
\tilde{\mu}_{|T-\lambda \mathbf{1}|}=\tilde{\mu}_{|T|} \boxplus \tilde{\mu}_{|\lambda| \mathbf{1}}=\tilde{\mu}_{|T|} \boxplus v,
$$

where $v=\frac{1}{2}\left(\delta_{-|\lambda|}+\delta_{|\lambda|}\right)$.

For $\beta>0$ define

$$
\Omega_{\beta}=\left\{w \in \mathrm{C}|0<| w \mid<\beta, \frac{5 \pi}{4}<\arg (w)<\frac{7 \pi}{4}\right\} .
$$

According to [4, Corollary 5.8], there is a $\beta>0$ such that for every $w \in \Omega_{\beta}$,

$$
\mathscr{R}_{\tilde{\mu}_{|T-\lambda| 1 \mid}}(w)=\mathscr{R}_{\tilde{\mu}_{|T|}}(w)+\mathscr{R}_{v}(w),
$$

where

$$
\mathscr{R}_{\nu}(w)=\frac{\sqrt{1+4|\lambda|^{2} w^{2}}-1}{2 w},
$$

and

$$
G_{\tilde{\mu}_{|T|}}(i s)=-i h(s), \quad s>0,
$$

whence

$$
\mathscr{R}_{\tilde{\mu}_{|T|}}(-i h(s))+\frac{1}{-i h(s)}=G_{\tilde{\mu}_{|T|}}^{\langle-1\rangle}(-i h(s))=i s, \quad s>0 .
$$

Take $s_{\lambda}>0$ such that for every $s>s_{\lambda},-i h(s) \in \Omega_{\beta}$. Then, when $s>s_{\lambda}$,

$$
\mathscr{R}_{\tilde{\mu}_{|T-\lambda| \mid}}(-i h(s))=i s+\frac{1}{i h(s)}+\frac{\sqrt{1-4|\lambda|^{2} h(s)^{2}}-1}{-2 i h(s)},
$$

implying that

$$
h(s)=h_{\lambda}\left(s+\frac{\sqrt{1-4|\lambda|^{2} h(s)^{2}}-1}{2 h(s)}\right) .
$$

That is, when $s>s_{\lambda}$ and

$$
t=s+\frac{\sqrt{1-4|\lambda|^{2} h(s)^{2}}-1}{2 h(s)},
$$

then $h(s)=h_{\lambda}(t)$.

Note that if

$$
t=s+\frac{\sqrt{1-4|\lambda|^{2} h(s)^{2}}-1}{2 h(s)},
$$

then $(s, t)$ satisfies the following equation:

$$
(s-t)\left(\frac{1}{h(s)}-s+t\right)=|\lambda|^{2} .
$$


In the following we will investigate this equation further.

Definition 4.3. Let $m, n \in \mathrm{N}$, and let $U$ be an open set in $\mathrm{R}^{m}$. A map $f: U \rightarrow \mathrm{R}^{n}$ is said to be analytic if it has a power series expansion in $m$ variables in a neighborhood of every $x \in U$.

We shall need the following two well-known lemmas about analytic functions of several variables:

Lemma 4.4. Let $U$ be a connected, open subset of $\mathbf{R}^{m}$. If $f, g: U \rightarrow \mathbf{R}^{n}$ are two analytic functions which coincide on a non-empty, open subset $V$ of $U$, then $f=g$.

Lemma 4.5. Let $U \subseteq \mathbf{R}^{m}$ be open and let $f: U \rightarrow \mathbf{R}^{m}$ be an analytic function for which the Jacobian $\mathscr{J}\left(x_{0}\right)=\operatorname{det} f^{\prime}\left(x_{0}\right)$ is non-zero for some $x_{0} \in U$. Then $f$ is one-to-one in some neighborhood $V$ of $x_{0}$, and the inverse of $\left.f\right|_{V}$ is analytic in a neighborhood of $f\left(x_{0}\right)$.

Lemma 4.6. Let $\mu$ be a probability measure on $[0, \infty)$, and define

$$
h(s)=\int_{0}^{\infty} \frac{s}{s^{2}+u^{2}} d \mu(u), \quad s>0 .
$$

Then $h$ is analytic on $(0, \infty)$. Moreover, if $\mu$ is not a Dirac measure, then for all $s>0$,

$$
0<h(s)<\frac{1}{s} \quad \text { and } \quad h^{\prime}(s)<\frac{h(s)}{s}-2 h(s)^{2} .
$$

Proof. Since

$$
h(s)=\frac{1}{2} \int_{0}^{\infty}\left(\frac{1}{s+i u}+\frac{1}{s-i u}\right) d \mu, \quad s>0,
$$

$h$ has a complex analytic extension

$$
\tilde{h}:\{z \in \mathrm{C} \mid \operatorname{Im} z>0\} \rightarrow \mathrm{C}
$$

given by the same formula. In particular, $h$ is an analytic function of $s \in(0, \infty)$. If $\mu$ is not a Dirac measure, then $\mu \neq \delta_{0}$, and so $h(s)>0$ for all $s>0$. Moreover,

$$
\operatorname{sh}(s)=\int_{0}^{\infty} \frac{s^{2}}{s^{2}+u^{2}} d \mu(u)<1, \quad s>0 .
$$


Finally, for $s>0$,

$$
\begin{aligned}
h(s)^{2} & =\int_{0}^{\infty} \int_{0}^{\infty} \frac{s}{s^{2}+u^{2}} \frac{s}{s^{2}+v^{2}} d \mu(u) d \mu(v) \\
& \leq \int_{0}^{\infty} \int_{0}^{\infty} \frac{1}{2}\left(\left(\frac{s}{s^{2}+u^{2}}\right)^{2}+\left(\frac{s}{s^{2}+v^{2}}\right)^{2}\right) d \mu(u) d \mu(v) \\
& =\int_{0}^{\infty} \frac{s^{2}}{\left(s^{2}+u^{2}\right)^{2}} d \mu(u) \\
& =\frac{1}{2}\left(\int_{0}^{\infty} \frac{s^{2}+u^{2}}{\left(s^{2}+u^{2}\right)^{2}} d \mu(u)+\int_{0}^{\infty} \frac{s^{2}-u^{2}}{\left(s^{2}+u^{2}\right)^{2}} d \mu(u)\right) \\
& =\frac{1}{2}\left(\frac{h(s)}{s}-h^{\prime}(s)\right) .
\end{aligned}
$$

Hence,

$$
h^{\prime}(s) \leq \frac{h(s)}{s}-2 h(s)^{2},
$$

and equality holds if and only if the product measure $\mu \otimes \mu$ is concentrated on the diagonal $\{(u, u) \mid u>0\}$. But this would imply that $\mu$ is a Dirac measure. Thus, if $\mu$ is not a Dirac measure, then

$$
h^{\prime}(s)<\frac{h(s)}{s}-2 h(s)^{2}, \quad s>0
$$

Lemma 4.7. Let $\mu$ be a probability measure on $[0, \infty)$ which is not a Dirac measure, and put

$$
\lambda_{1}(\mu)=\left(\int_{0}^{\infty} \frac{1}{u^{2}} d \mu(u)\right)^{-\frac{1}{2}} \quad \text { and } \quad \lambda_{2}(\mu)=\left(\int_{0}^{\infty} u^{2} d \mu(u)\right)^{\frac{1}{2}},
$$

with the convention that $\infty^{-\frac{1}{2}}=0$. Then $0 \leq \lambda_{1}(\mu)<\lambda_{2}(\mu) \leq \infty$.

Proof. Clearly, $\lambda_{1}(\mu)<\infty$, and since $\mu \neq \delta_{0}, \lambda_{2}(\mu)>0$. The lemma is then trivially true if $\lambda_{1}(\mu)=0$ or $\lambda_{2}(\mu)=+\infty$. Thus, we can assume that $\lambda_{1}(\mu), \lambda_{2}(\mu) \in(0, \infty)$. Then, by the Schwartz inequality,

$$
\begin{aligned}
\frac{\lambda_{2}(\mu)}{\lambda_{1}(\mu)} & =\left(\int_{0}^{\infty} u^{2} d \mu(u)\right)^{\frac{1}{2}}\left(\int_{0}^{\infty} \frac{1}{u^{2}} d \mu(u)\right)^{\frac{1}{2}} \\
& \geq \int_{0}^{\infty} u \frac{1}{u} d \mu(u)=1,
\end{aligned}
$$


and equality holds if and only if for some $c \in(0, \infty), \frac{1}{u}=c u$ holds for $\mu$-a.e. $u \in[0, \infty)$. However, this can not be the case when $\mu$ is not a Dirac measure.

Lemma 4.8. Let $\mu, \lambda_{1}(u)$ and $\lambda_{2}(\mu)$ be as in Lemma 4.7, and let $h$ be as in Lemma 4.6. Then put

$$
k(s, t)=(s-t)\left(\frac{1}{h(s)}-s+t\right), \quad s>0, t \in \mathbf{R} .
$$

Then $k$ is an analytic function on $(0, \infty) \times \mathbf{R}$. Moreover, for $t>0$ the map $s \mapsto k(s, t)$ is a strictly increasing bijection of $(t, \infty)$ onto $(0, \infty)$, and for $t=0$ the map $s \mapsto k(s, t)$ is a strictly increasing bijection of $(0, \infty)$ onto $\left(\lambda_{1}(\mu)^{2}, \lambda_{2}(\mu)^{2}\right)$.

Proof. Clearly, $k$ is analytic. Moreover,

$$
\frac{\partial k}{\partial s}(s, t)=\frac{1}{h(s)}-(s-t)\left(2+\frac{h^{\prime}(s)}{h(s)^{2}}\right) .
$$

For $s \in(0, \infty)$, we get from Lemma 4.6 that

$$
\frac{\partial k}{\partial s}(s, 0)=\frac{s}{h(s)^{2}}\left(\frac{h(s)}{s}-2 h(s)^{2}-h^{\prime}(s)\right)>0,
$$

and

$$
\frac{\partial k}{\partial s}(s, s)=\frac{1}{h(s)}>s .
$$

Since the right-hand side of (4.4) is an affine function of $t \in \mathrm{R}$, it follows that

$$
\frac{\partial k}{\partial s}(s, t)>t, \quad s>0, t \in[0, s] .
$$

Hence, $s \mapsto k(s, t)$ is a strictly increasing function of $s \in(t, \infty)$ for every $t \in[0, \infty)$. For $s>t>0$,

$$
k(s, t)=\int_{t}^{s} \frac{\partial k}{\partial s^{\prime}}\left(s^{\prime}, t\right) d s^{\prime}>\int_{t}^{s} t d s^{\prime}=t(s-t) .
$$

Hence, when $t>0$,

$$
\lim _{s \rightarrow \infty} k(s, t)=\infty,
$$

and

$$
\lim _{s \rightarrow t+} k(s, t)=k(t, t)=0 .
$$

Thus, $s \mapsto k(s, t)$ is a bijection of $(t, \infty)$ onto $(0, \infty)$. 
Next, consider the case $t=0$. We have already seen that $s \mapsto k(s, 0)$ is strictly increasing on $(0, \infty)$. Note that for $s>0$,

$$
k(s, 0)=\frac{1-s h(s)}{h(s) / s}=\frac{n(s)}{d(s)}
$$

where

$$
n(s)=\int_{0}^{\infty} \frac{u^{2}}{s^{2}+u^{2}} d \mu(u) \quad \text { and } \quad d(s)=\int_{0}^{\infty} \frac{1}{s^{2}+u^{2}} d \mu(u) .
$$

By the monotone convergence theorem,

$$
\begin{gathered}
\lim _{s \rightarrow 0+} n(s)=1, \\
\lim _{s \rightarrow 0+} d(s)=\int_{0}^{\infty} \frac{1}{u^{2}} d \mu(u)=\frac{1}{\lambda_{1}(\mu)^{2}}, \\
\lim _{s \rightarrow \infty} s^{2} n(s)=\int_{0}^{\infty} u^{2} d \mu(u)=\lambda_{2}(\mu)^{2},
\end{gathered}
$$

and

$$
\lim _{s \rightarrow \infty} s^{2} d(s)=1
$$

Hence,

$$
\lim _{s \rightarrow 0+} k(s, 0)=\lambda_{1}(\mu)^{2},
$$

and

$$
\lim _{s \rightarrow \infty} k(s, 0)=\lambda_{2}(\mu)^{2}
$$

This shows that $s \mapsto k(s, 0)$ is a bijection of $(0, \infty)$ onto $\left(\lambda_{1}(\mu)^{2}, \lambda_{2}(\mu)^{2}\right)$.

Definition 4.9. Let $\mu, \lambda_{1}(u)$ and $\lambda_{2}(\mu)$ be as in Lemma 4.7, let $h$ be as in Lemma 4.6, and let $k$ be as in Lemma 4.8. For $\lambda, t \in(0, \infty)$, let $s(\lambda, t)$ denote the unique solution $s \in(t, \infty)$ to the equation $k(s, t)=\lambda^{2}$ (cf. Lemma 4.8), and for $\lambda \in\left(\lambda_{1}(\mu), \lambda_{2}(\mu)\right)$, let $s(\lambda, 0)$ denote the unique solution $s \in(0, \infty)$ to the equation $k(s, 0)=\lambda^{2}$.

Lemma 4.10. The function $(\lambda, t) \mapsto s(\lambda, t)$ is analytic in $(0, \infty) \times(0, \infty)$. Moreover, for $\lambda \in\left(\lambda_{1}(\mu), \lambda_{2}(\mu)\right)$,

$$
\lim _{t \rightarrow 0+} s(\lambda, t)=s(\lambda, 0) .
$$

ProOF. Let

$$
\Omega=\left\{(s, t) \in \mathrm{R}^{2} \mid 0<t<s\right\} .
$$


According to Lemma 4.8, $k$ is a strictly positive, analytic function in $\Omega$. Let

$$
F(s, t)=(\sqrt{k(s, t)}, t), \quad(s, t) \in \Omega .
$$

Then $F$ is analytic in $\Omega$, and by Lemma $4.8, F$ is a one-to-one map of $\Omega$ onto $(0, \infty) \times(0, \infty)$. Moreover, its inverse $F^{-1}:(0, \infty) \times(0, \infty) \rightarrow \Omega$ is given by

$$
F^{-1}(\lambda, t)=(s(\lambda, t), t), \quad s, t>0 .
$$

The Jacobian of $F$ is

$$
\mathscr{J}(F)(s, t)=\frac{\partial}{\partial s} \sqrt{k(s, t)}=\frac{1}{2 \sqrt{k(s, t)}} \frac{\partial k}{\partial s}(s, t),
$$

which by (4.5) is strictly positive for all $(s, t) \in \Omega$. Hence, by Lemma $4.5, F^{-1}$ is analytic in $(0, \infty) \times(0, \infty)$. In particular, $s(\lambda, t)$ is analytic in $(0, \infty) \times$ $(0, \infty)$.

Now, let $\lambda_{0} \in\left(\lambda_{1}(\mu), \lambda_{2}(\mu)\right)$ and put $s_{0}=s\left(\lambda_{0}, 0\right)$. Then $k\left(s_{0}, 0\right)=\lambda_{0}^{2}$, and by the proof of Lemma 4.8, $\frac{\partial k}{\partial s}\left(s_{0}, 0\right)>0$. Let

$$
F_{0}(s, t):=(\sqrt{k(s, t)}, t)
$$

$F_{0}$ is then analytic in some neighborhood $U_{0}$ of $\left(s_{0}, 0\right)$. Moreover, $\mathscr{J}\left(F_{0}\right)\left(s_{0}, 0\right)$ $\neq 0$, and therefore, by Lemma $4.5, F_{0}$ has an analytic inverse $F_{0}^{-1}$ in a neighborhood $V_{0}$ of $F_{0}\left(s_{0}, 0\right)=\left(\lambda_{0}, 0\right)$. Clearly, $F_{0}^{-1}(\lambda, t)=F^{-1}(\lambda, t)$, whenever $(\lambda, t) \in V_{0} \cap[(0, \infty) \times(0, \infty)]$, and $F_{0}^{-1}(\lambda, t) \in \Omega$.

Note that

$$
\lim _{t \rightarrow 0+} F_{0}^{-1}\left(\lambda_{0}, t\right)=F_{0}^{-1}\left(\lambda_{0}, 0\right)=\left(s_{0}, 0\right)
$$

and since the second coordinate of $F_{0}^{-1}\left(\lambda_{0}, t\right)$ is $t$, we conclude that $F_{0}^{-1}\left(\lambda_{0}, t\right)$ $\in \Omega$, eventually as $t \rightarrow 0+$. Hence,

$$
\left(s_{0}, 0\right)=\lim _{t \rightarrow 0+} F_{0}^{-1}\left(\lambda_{0}, t\right)=\lim _{t \rightarrow 0+} F^{-1}\left(\lambda_{0}, t\right)=\lim _{t \rightarrow 0+}\left(s\left(\lambda_{0}, t\right), t\right),
$$

and therefore,

$$
\lim _{t \rightarrow 0+} s\left(\lambda_{0}, t\right)=s_{0}=s\left(\lambda_{0}, 0\right) .
$$

REMARK 4.11. We get from Lemma 4.10 that

$$
\lim _{t \rightarrow 0+} s(\lambda, t)=0, \quad 0<\lambda \leq \lambda_{1}(\mu)
$$


and

$$
\lim _{t \rightarrow 0+} s(\lambda, t)=+\infty, \quad \lambda \geq \lambda_{2}(\mu)
$$

Indeed, for fixed $t>0, \lambda \mapsto s(\lambda, t)$ is a monotonically increasing function of $\lambda$. Hence, if $0<\lambda \leq \lambda_{1}(\mu)$, then

$$
\limsup _{t \rightarrow 0+} s(\lambda, t) \leq \limsup _{t \rightarrow 0+} s\left(\lambda^{\prime}, t\right)=s\left(\lambda^{\prime}, 0\right),
$$

for all $\lambda^{\prime} \in\left(\lambda_{1}(\mu), \lambda_{2}(\mu)\right)$.

But $\lambda^{\prime} \mapsto s\left(\lambda^{\prime}, 0\right)$ is the inverse function of $s \mapsto \sqrt{k(s, 0)}$, and hence $\lambda^{\prime} \mapsto s\left(\lambda^{\prime}, 0\right)$ is a bijection of $\left(\lambda_{1}(\mu), \lambda_{2}(\mu)\right)$ onto $(0, \infty)$. It follows that $\lim \sup _{t \rightarrow 0+} s(\lambda, t)=0$, and this proves (4.9).

For $\lambda \geq \lambda_{2}(\mu)$, a similar argument shows that $\liminf _{t \rightarrow 0+} s(\lambda, t)=+\infty$, and this proves (4.10).

LeMmA 4.12. Let $\lambda>0$. Then

(i) $\lim _{t \rightarrow \infty}(s(\lambda, t)-t)=0$, and

(ii) there exists a $t_{\lambda}>0$ such that when $t>t_{\lambda}$ and $s=s(\lambda, t)$, then

$$
t=s+\frac{\sqrt{1+4 \lambda^{2} h(s)^{2}}-1}{2 h(s)} .
$$

Proof. Fix $t>0$, and put $s=s(\lambda, t)$. Then by Definition 4.9, $s>t$ and $k(s, t)=\lambda^{2}$. According to (4.6), $k(s, t)>t(s-t)$. Hence,

$$
0<s-t<\frac{\lambda^{2}}{t} \text {. }
$$

This proves (i). With $s$ and $t$ as above,

$$
\lambda^{2}=k(s, t)=(s-t)\left(\frac{1}{h(s)}-s+t\right) .
$$

Solving this equation for $t$, we get that $t$ is one of the two numbers

$$
t_{ \pm}=s-\frac{1}{h(s)} \pm \frac{\sqrt{1+4 \lambda^{2} h(s)^{2}}}{2 h(s)} .
$$

If $t=t_{-}$, then

$$
s-t>\frac{1}{2 h(s)},
$$


and since $\frac{1}{h(s)} \rightarrow \infty$ as $s \rightarrow \infty$, this can not hold for large $t$ because of (i). Hence, $t=t_{+}$for $t$ sufficiently large.

Combining the previous lemmas we get:

Proposition 4.13. Let $T \in \tilde{M}$ be an $R$-diagonal element, let $\lambda \in \mathrm{C} \backslash\{0\}$, and define $h(s)$ and $h_{\lambda}(s)$ as in Lemma 4.2. Let $\mu=\mu_{|T|}$, and let $s(|\lambda|, t)$ be as in Definition 4.9. Then

$$
h_{\lambda}(s(|\lambda|, t))=h(t), \quad t>0 .
$$

Proof. According to Lemma 4.12, if $t>t_{|\lambda|}$ and $s=s(|\lambda|, t)$, then

$$
t=s+\frac{\sqrt{1+4 \lambda^{2} h(s)^{2}}-1}{2 h(s)} .
$$

Since $s(|\lambda|, t)>t$, we infer from Lemma 4.2 that for $t$ sufficiently large,

$$
h_{\lambda}(t)=h(s(|\lambda|, t)) \text {. }
$$

Hence, by Lemma 4.4 and Lemma 4.10, the same formula holds for all $t>0$.

Lemma 4.14. Let $T$ be an unbounded $R$-diagonal element in $\mathcal{M}^{\Delta}$, let $\lambda \in \mathrm{C} \backslash\{0\}$, and let $t>0$. With $\mu=\mu_{|T|}$ and $s(|\lambda|, t)$ as in Definition 4.9 we then have:

$\Delta\left((T-\lambda \mathbf{1})^{*}(T-\lambda \mathbf{1})+t^{2} \mathbf{1}\right)=\frac{|\lambda|^{2}}{|\lambda|^{2}+(s(|\lambda|, t)-t)^{2}} \Delta\left(T^{*} T+s(|\lambda|, t)^{2} \mathbf{1}\right)$.

Proof. Since $T$ is $R$-diagonal, $T \underset{* \mathscr{D}}{\sim} c T$ for all $c \in \mathrm{T}$. Hence, the left-hand side of (4.11) depends only on $|\lambda|$. It therefore suffices to consider only the case $\lambda>0$. For $\lambda, t>0$, let

$$
H(t)=\frac{1}{2} \log \Delta\left(T^{*} T+t^{2} \mathbf{1}\right)
$$

and

$$
H_{\lambda}(t)=\frac{1}{2} \log \Delta\left((T-\lambda \mathbf{1})^{*}(T-\lambda \mathbf{1})+t^{2} \mathbf{1}\right) .
$$

Then with $\mu_{\lambda}=\mu_{|T-\lambda \mathbf{1}|}$,

$$
H(t)=\frac{1}{2} \int_{0}^{\infty} \log \left(u^{2}+t^{2}\right) d \mu(u),
$$

and

$$
H_{\lambda}(t)=\frac{1}{2} \int_{0}^{\infty} \log \left(u^{2}+t^{2}\right) d \mu_{\lambda}(u)
$$


Since $T$ and $T-\lambda \mathbf{1}$ belong to $\mathcal{M}^{\Delta}, H$ and $H_{\lambda}$ take values in R. Moreover, $H$ and $H_{\lambda}$ are differentiable with derivatives $H^{\prime}(t)=h(t)$ and $H_{\lambda}^{\prime}(t)=h_{\lambda}(t)$. Also, since $T \in \mathscr{M}^{\Delta}$,

$$
\lim _{t \rightarrow \infty}(H(t)-\log t)=\frac{1}{2} \lim _{t \rightarrow \infty} \int_{0}^{\infty} \log \left(1+\frac{u^{2}}{t^{2}}\right) d \mu(u)=0,
$$

and similarly

$$
\lim _{t \rightarrow \infty}\left(H_{\lambda}(t)-\log t\right)=0 .
$$

Fix $\lambda>0$ and $t_{0}>0$. There is a constant $C$ such that

$$
H_{\lambda}(t)=\int_{t_{0}}^{t} h_{\lambda}\left(t^{\prime}\right) d t^{\prime}+C
$$

Moreover, according to Proposition 4.13,

$$
h_{\lambda}(t)=h(s(\lambda, t)), \quad t>0 .
$$

Put $s(t)=s(\lambda, t)$ and $u(t)=t-s(t)$. Then $s(t)+u(t)=t$ and $s^{\prime}(t)+u^{\prime}(t)=$ 1. Moreover, by Definition 4.9,

$$
(s(t)-t)\left(\frac{1}{h(s(t))}-s(t)+t\right)=\lambda^{2} .
$$

Hence,

$$
u(t)\left(\frac{1}{h(s(t))}-u(t)\right)=\lambda^{2}
$$

implying that

$$
h(s(t))=\frac{u(t)}{\lambda^{2}+u(t)^{2}} .
$$

It follows that

$$
\begin{aligned}
\int_{t_{0}}^{t} h_{\lambda}(v) d v \\
\quad=\int_{t_{0}}^{t} h(s(v))\left(s^{\prime}(v)+u^{\prime}(v)\right) d v \\
\quad=\int_{t_{0}}^{t}\left(h(s(v)) s^{\prime}(v)+\frac{u(v)}{\lambda^{2}+u(v)^{2}} u^{\prime}(v)\right) d v \\
\quad=H(s(t))-H\left(s\left(t_{0}\right)\right)+\frac{1}{2} \log \left(\frac{\lambda^{2}}{\lambda^{2}+u(t)^{2}}\right)+\frac{1}{2} \log \left(\frac{\lambda^{2}+u\left(t_{0}\right)^{2}}{\lambda^{2}}\right) .
\end{aligned}
$$


Hence,

$$
H_{\lambda}(t)=H(s(t))+\frac{1}{2} \log \left(\frac{\lambda^{2}}{\lambda^{2}+(s(t)-t)^{2}}\right)+C^{\prime},
$$

for a constant $C^{\prime}$. Recall that $s(t)-t \rightarrow 0$ as $t \rightarrow \infty$ (cf. Lemma 4.12). It then follows from (4.12) and (4.13) that $C^{\prime}$ must be 0 . This finally shows us that

$$
\exp \left(2 H_{\lambda}(t)\right)=\frac{\lambda^{2}}{\lambda^{2}+(s(t)-t)^{2}} \exp (2 H(t))
$$

and this proves (4.11).

THEOREM 4.15. Let $T \in \mathscr{M}^{\Delta}$ be $R$-diagonal, let $\mu=\mu_{|T|}$, and let $s(|\lambda|, 0)$ be as in Definition 4.9 .

(i) If $\lambda_{1}(\mu)<|\lambda|<\lambda_{2}(\mu)$, then

$$
\Delta(T-\lambda \mathbf{1})=\left(\frac{|\lambda|^{2}}{|\lambda|^{2}+s(|\lambda|, 0)^{2}} \Delta\left(T^{*} T+s(|\lambda|, 0)^{2} \mathbf{1}\right)\right)^{\frac{1}{2}} .
$$

(ii) If $|\lambda| \leq \lambda_{1}(\mu)$, then $\Delta(T-\lambda \mathbf{1})=\Delta(T)$.

(iii) If $|\lambda| \geq \lambda_{2}(\mu)$, then $\Delta(T-\lambda \mathbf{1})=|\lambda|$.

Proof. The theorem is obviously true for $\lambda=0$. Moreover, as in the proof of Lemma 4.14, it suffices to consider the case $\lambda>0$. Note that

$$
\Delta(T-\lambda \mathbf{1})^{2}=\lim _{t \rightarrow 0+} \Delta\left((T-\lambda \mathbf{1})^{*}(T-\lambda \mathbf{1})+t^{2} \mathbf{1}\right) .
$$

Hence, (i) follows from Lemma 4.10 and Lemma 4.14. If $0<\lambda \leq \lambda_{1}(\mu)$, then by Remark 4.11, $\lim _{t \rightarrow 0+} s(\lambda, t)=0$. Hence, (ii) also follows from Lemma 4.14. Now suppose $\lambda \geq \lambda_{2}(\mu)$. Then $s(\lambda, t) \rightarrow \infty$ as $t \rightarrow 0+$. The right-hand side of (4.11) is equal to

$$
\frac{\lambda^{2} s(\lambda, t)^{2}}{\lambda^{2}+(s(\lambda, t)-t)^{2}} \frac{\Delta\left(T^{*} T-s(\lambda, t)^{2} \mathbf{1}\right)}{s(\lambda, t)^{2}},
$$

where the first factor converges to $\lambda^{2}$ as $t \rightarrow 0+$, and the second factor converges to 1 (cf. (4.12)). (iii) now follows from (4.11) and (4.14).

REMARK 4.16. Note that

$$
\lambda_{2}(\mu)=\left(\int_{0}^{\infty} u^{2} d \mu_{|T|}(u)\right)^{\frac{1}{2}}=\|T\|_{2}
$$

and

$$
\lambda_{1}(\mu)=\left(\int_{0}^{\infty} u^{-2} d \mu_{|T|}(u)\right)^{-\frac{1}{2}}=\left\|T^{-1}\right\|_{2}^{-1},
$$


where $\left\|T^{-1}\right\|_{2}:=+\infty$ in case $\operatorname{ker}(T) \neq 0$.

THEOREM 4.17. Let $T$ be an $R$-diagonal element in $\mathcal{M}^{\Delta}$ with Brown measure $\mu_{T}$, and suppose $\mu_{|T|}$ is not a Dirac measure.

(a) If $\operatorname{ker}(T)=0$, then

$$
\operatorname{supp}\left(\mu_{T}\right)=\left\{\lambda \in \mathrm{C}\left|\left\|T^{-1}\right\|_{2}^{-1} \leq\right| \lambda \mid \leq\|T\|_{2}\right\} .
$$

Moreover, the S-transform of $\mu_{|T|^{2}}$ is well-defined and strictly increasing on $(-1,0)$ with

$$
\mathscr{S}_{\mu_{|T|^{2}}}((-1,0))=\left(\|T\|_{2}^{-2},\left\|T^{-1}\right\|_{2}^{2}\right),
$$

and $\mu_{T}$ is the unique probability measure on $\mathrm{C}$ which is invariant under rotations and satisfies

$$
\mu_{T}\left(B\left(0, \mathscr{S}_{\mu_{|T|^{2}}}(t-1)^{-\frac{1}{2}}\right)\right)=t, \quad 0<t<1 .
$$

(b) If $\operatorname{ker}(T) \neq 0$, let $P$ denote the projection onto $\operatorname{ker}(T)$. Then

$$
\operatorname{supp}\left(\mu_{T}\right)=\left\{\lambda \in \mathrm{C}|| \lambda \mid \leq\|T\|_{2}\right\} .
$$

Moreover, the S-transform of $\mu_{|T|^{2}}$ is well-defined and strictly increasing on $(\tau(P)-1,0)$ with

$$
\mathscr{S}_{\mu_{|T|^{2}}}((\tau(P)-1,0))=\left(\|T\|_{2}^{-2}, \infty\right),
$$

and $\mu_{T}$ is the unique probability measure on $\mathrm{C}$ which is invariant under rotations and satisfies

$$
\mu_{T}\left(B\left(0, \mathscr{S}_{\mu_{|T|^{2}}}(t-1)^{-\frac{1}{2}}\right)\right)=t, \quad \tau(P)<t<1 .
$$

Proof. By definition, $d \mu_{T}(\lambda)=\frac{1}{2 \pi} \nabla^{2}(\log \Delta(T-\lambda \mathbf{1})) d \lambda$ (in the distribution sense). Hence, $\mu_{T}$ can be determined from Theorem 4.15 in the same way as $[9$, Theorem 4.4.] is obtained from $[9,(4.5)]$ :

Using the same notation as in [9], we define functions $f, g:(0, \infty) \rightarrow \mathbf{R}$ by

$$
f(v)=\int_{0}^{\infty} \frac{1}{1+v^{2} w^{2}} d \mu_{|T|}(w),
$$

and

$$
g(v)=\frac{1-f(v)}{v^{2} f(v)} .
$$


Moreover, for $\lambda \in\left(\left\|T^{-1}\right\|_{2}^{-2},\|T\|_{2}^{2}\right)$, let $v(\lambda)$ denote the unique $v \in(0, \infty)$ such that $g(v)=\lambda^{2}$. Then, in our notation,

$$
f(v)=\tau\left(\left(\mathbf{1}+v^{2} T^{*} T\right)^{-1}\right)=v^{-1} h\left(v^{-1}\right),
$$

and

$$
g(v)=v^{-1}\left(\frac{1}{h\left(v^{-1}\right)}-v^{-1}\right)=k\left(v^{-1}, 0\right) .
$$

Hence,

$$
v(\lambda)=\frac{1}{s(\lambda, 0)}
$$

and it follows that the formula (4.15) in [9],

$$
\log \Delta(T-\lambda \mathbf{1})=\frac{1}{2} \int_{0}^{\infty} \log \left(1+v^{2} w^{2}\right) d \mu_{|T|}(w)+\frac{1}{2} \log \left(\frac{\lambda^{2}}{1+v^{2} \lambda^{2}}\right),
$$

$\lambda \in\left(\left\|T^{-1}\right\|_{2}^{-2},\|T\|_{2}^{2}\right)$, is equivalent to the one in Theorem 4.15 (i). The rest of the proof of Theorem 4.17 is identical to the second part of the proof of [9, Theorem 4.4], since boundedness of $T$ is not a necessary assumption in the latter.

Remark 4.18. Let $T \in \mathscr{M}^{\Delta}$ be $R$-diagonal. Then $\operatorname{supp}\left(\mu_{T}\right) \subseteq \sigma(T)$, and according to Theorem 4.17 ,

$$
\operatorname{supp}\left(\mu_{T}\right)=\left\{\lambda \in \mathrm{C}\left|\left\|T^{-1}\right\|_{2}^{-1} \leq\right| \lambda \mid \leq\|T\|_{2}\right\} .
$$

Moreover, by arguments similar to the ones given in [9, proof of Proposition 4.6], one can show that

(a) if $0<|\lambda|<\left\|T^{-1}\right\|_{2}^{-1}$, then $\lambda \in \sigma(T)$ iff $T$ does not have a bounded inverse, and

(b) if $|\lambda|>\|T\|_{2}$, then $\lambda \in \sigma(T)$ iff $T$ is not bounded.

\section{Properties of $z=x y^{-1}$}

Let $\mathscr{M}=L\left(\mathrm{~F}_{4}\right)$ be the von Neumann algebra associated with the free group on 4 generators. According to [17] or [18], $\mathcal{M}$ is a $\mathrm{II}_{1}$-factor generated by a semicircular system $\left(s_{1}, s_{2}, s_{3}, s_{4}\right)$, i.e. the $s_{i}$ 's are freely independent selfadjoint elements w.r.t. the unique tracial state $\tau$ on $\mathscr{M}$, and $s_{i}$ has distribution

$$
d \mu_{s_{i}}(t)=\frac{1}{2 \pi} \sqrt{4-t^{2}} 1_{[-2,2]}(t) d t, \quad 1 \leq i \leq 4 .
$$


Put

$$
x=\frac{s_{1}+i s_{2}}{\sqrt{2}} \quad \text { and } \quad y=\frac{s_{3}+i s_{4}}{\sqrt{2}} .
$$

Then $\mathcal{M}=W^{*}(x, y)$, and $(x, y)$ is a circular system in the sense of [18]. Also, by [18], $|y|$ has the distribution

$$
d \mu_{|y|}(t)=\frac{2}{\pi} \sqrt{4-t^{2}} 1_{[0,2]}(t) d t .
$$

In particular, $\operatorname{ker}(y)=0$. In this section we will study the unbounded operator

$$
z=x y^{-1}
$$

as well as its powers $z^{n}, n=2,3, \ldots$ We will need the following simple observation:

LEMMA 5.1. Let $\left(\mu_{n}\right)_{n=1}^{\infty}$ and $\mu$ be probability measures on $\mathrm{R}$ with densities $\left(f_{n}\right)_{n=1}^{\infty}$ and $f$, respectively, w.r.t. Lebesgue measure. If $f_{n} \stackrel{n \rightarrow \infty}{\longrightarrow} f$ a.e. w.r.t. Lebesgue measure, then $\mu_{n} \stackrel{n \rightarrow \infty}{\longrightarrow} \mu$ weakly.

Proof. Recall that $\mu_{n} \stackrel{n \rightarrow \infty}{\longrightarrow} \mu$ weakly iff for all $\phi \in C_{0}(\mathrm{R})$,

$$
\lim _{n \rightarrow \infty} \int_{\mathrm{R}} \phi d \mu_{n}=\int_{\mathrm{R}} \phi d \mu .
$$

Clearly, it suffices to consider $\phi \in C_{0}$ (R) with $0 \leq \phi \leq 1$, and for such $\phi$, (5.1) follows for such $\phi$ by application of Fatou's Lemma to each of the sequences of integrals $\left(\int_{\mathrm{R}} \phi f_{n} d m\right)_{n=1}^{\infty}$ and $\left(\int_{\mathrm{R}}(1-\phi) f_{n} d m\right)_{n=1}^{\infty}$.

TheOREM 5.2. Let $(\mathcal{M}, \tau)$ and $z=x y^{-1}$ be as above.

(a) $z$ is an unbounded, $R$-diagonal operator.

(b) The distribution of $z$ is given by

$$
d \mu_{|z|}(t)=\frac{2}{\pi} \frac{1}{1+t^{2}} 1_{(0, \infty)}(t) d t .
$$

(c) For $p \in(0,1), z, z^{-1} \in L^{p}(\mathscr{M}, \tau)$, and

$$
\|z\|_{p}^{p}=\left\|z^{-1}\right\|_{p}^{p}=\left[\cos \left(\frac{p \pi}{2}\right)\right]^{-1}<\infty .
$$

(d) $z, z^{-1} \in \mathcal{M}^{\Delta}$, and the Brown measure of $z$ is given by

$$
d \mu_{z}(s)=\frac{1}{\pi\left(1+|s|^{2}\right)^{2}} d s
$$

where $d s=d \operatorname{Re} s d \operatorname{Im} s$ is Lebesgue measure on C. 
Proof. (a) Let $x=u|x|$ and $y=v|y|$ be the polar decompositions of $x$ and $y$. Then, according to [18], $u,|x|, v$ and $|y|$ are $*$-free elements, and $u$ and $v$ are Haar unitaries. In particular, $x$ and $y$ are $R$-diagonal and so is $y^{-1}$ (cf. Proposition 3.6). Moreover, $y^{-1}$ has polar decomposition

$$
y^{-1}=v^{*}\left(v|y|^{-1} v^{*}\right)=v^{*}\left|y^{*}\right|^{-1},
$$

which implies that $y^{-1}$ is affiliated with $W^{*}(y)$. Hence, $x$ and $y^{-1}$ are $*$-free, and it follows from Proposition 3.8 that $z=x y^{-1}$ is $R$-diagonal with

$$
\mathscr{S}_{\mu_{|z|^{2}}}(t)=\mathscr{S}_{\mu_{|x|^{2}}}(t) \mathscr{S}_{\mu_{\left|y^{-1}\right|^{2}}}(t), \quad t \in(-1,0) .
$$

The distribution of $|x|^{2}$ has density

$$
d \mu_{|x|^{2}}(t)=\frac{1}{2 \pi} \sqrt{\frac{4-t}{t}} 1_{(0,4)}(t) d t,
$$

and thus $\mathscr{S}_{\mu_{|x|^{2}}}$ is given by

$$
\mathscr{S}_{\mu_{|x|^{2}}}(t)=\frac{1}{1+t}
$$

for all $t$ in a neighborhood of $(-1,0)$ (cf. [9, example 5.2]). Since $\left|y^{-1}\right|=$ $\left|y^{*}\right|^{-1} \underset{* \mathscr{D}}{\sim}|y|^{-1} \underset{* \mathscr{D}}{\sim}|x|^{-1}$, we get from Proposition 3.13 that

$$
\mathscr{S}_{\mu_{\mid y}-\left.1\right|^{2}}(t)=\frac{1}{\mathscr{S}_{\mu_{|x|^{2}}}(-1-t)}=-t, \quad t \in(-1,0) .
$$

Then

$$
\mathscr{S}_{\mu_{|z|^{2}}}(t)=-\frac{t}{1+t}, \quad t \in(-1,0),
$$

and

$$
\chi_{\mu_{\left.||\right|^{2}}}(t)=\frac{t}{1+t} \mathscr{S}_{\mu_{|z|^{2}}}(t)=-\left(\frac{t}{1+t}\right)^{2}, \quad t \in(-1,0) .
$$

The inverse function of $\chi_{\left.\mu_{|z|}\right|^{2}}$ is then

$$
\psi_{\mu_{|z|^{2}}}(u)=\frac{-\sqrt{-u}}{1+\sqrt{-u}}, \quad u \in(-\infty, 0),
$$

and it follows that

$$
G_{\mu_{|z|^{2}}}(\lambda)=\frac{1}{\lambda}\left(1+\psi_{\mu_{|z|^{2}}}\left(\frac{1}{\lambda}\right)\right)=\frac{1}{\lambda-\sqrt{-\lambda}}, \quad \lambda<0 .
$$


Let $\sqrt{w}$ denote the principal value of the square root of $w$ for $w \in \mathrm{C} \backslash(\infty, 0]$. Then both sides of (5.6) are analytic in $C \backslash[0, \infty)$. Thus, (5.6) holds for all $\lambda \in \mathrm{C} \backslash[0, \infty)$, and it follows that for $t>0$,

(5.7) $-\frac{1}{\pi} \lim _{u \rightarrow 0+} \operatorname{Im} G_{\mu_{|z|^{2}}}(t+i u)=-\frac{1}{\pi} \operatorname{Im}\left(\frac{1}{t+i \sqrt{t}}\right)=\frac{1}{\pi} \frac{1}{\sqrt{t}(t+1)}$.

For $\beta \in(0,1)$,

$$
\int_{0}^{\infty} \frac{t^{\beta-1}}{1+t} d t=\frac{\pi}{\sin (\beta \pi)}
$$

(cf. [12, p. 592, formula 613]). The right-hand side of (5.7) therefore defines the density of a probability measure, and then, by Lemma 5.1, the probability measures

$$
\frac{1}{\pi} \operatorname{Im} G_{\mu_{|k|^{2}}}(t+i u) d t, \quad u>0,
$$

converge weakly to

$$
\frac{1}{\pi} \frac{1}{\sqrt{t}(t+1)} 1_{(0, \infty)}(t) d t
$$

as $u \rightarrow 0+$. Hence, by the inverse Stieltjes transform, $d \mu_{|z|^{2}}(t)$ is given by (5.9), and then

$$
d \mu_{|z|}(t)=\frac{2}{\pi} \frac{1}{1+t^{2}} 1_{(0, \infty)}(t) d t
$$

This proves (a) and (b).

In order to prove (c), note that according to (5.8),

$$
\tau\left(|z|^{p}\right)=\frac{2}{\pi} \int_{0}^{\infty} \frac{t^{p}}{1+t^{2}} d t=\frac{1}{\pi} \int_{0}^{\infty} \frac{w^{\frac{p-1}{2}}}{1+w} d w=\left[\sin \left(\frac{\pi(p+1)}{2}\right)\right]^{-1},
$$

proving (c). Since $L^{p}(\mathscr{M}, \tau) \subseteq \mathscr{M}^{\Delta}, p>0, z, z^{-1} \in \mathscr{M}^{\Delta}$. According to Theorem 4.17, $\mu_{z}$ is then the unique probability measure on $C$ which is invariant under rotations and satisfies

$$
\mu_{z}\left(B\left(0, \mathscr{S}_{\mu_{|z|^{2}}}(t-1)^{-\frac{1}{2}}\right)\right)=t, \quad 0<t<1 .
$$

Then by (5.5),

$$
\mu_{z}\left(B\left(0, \sqrt{\frac{t}{1-t}}\right)\right)=t, \quad 0<t<1,
$$


that is,

$$
\mu_{z}(B(0, r))=\frac{r^{2}}{1+r^{2}}, \quad r>0 .
$$

Hence, $\frac{d}{d r} \mu_{z}(B(0, r))=\frac{2 r}{\left(1+r^{2}\right)^{2}}$, and combining this with the fact that $\mu_{z}$ is invariant under rotations, we find that $\mu_{z}$ has density w.r.t. Lebesgue measure on $\mathrm{C}$ given by

$$
\frac{1}{2 \pi r} \frac{2 r}{\left(1+r^{2}\right)^{2}}=\frac{1}{\pi} \frac{1}{\left(1+r^{2}\right)^{2}}, \quad r>0,
$$

where $r=|s|, s \in \mathrm{C} \backslash\{0\}$. This proves (d).

LEMMA 5.3. Let $\mu$ be a probability measure on $[0, \infty)$ and, as in section 5 , put

$$
h(s)=\int_{0}^{\infty} \frac{s}{s^{2}+u^{2}} d \mu(u), \quad s \in(0, \infty) .
$$

Then for $0<p<2$,

$$
\int_{0}^{\infty} u^{-p} d \mu(u)=\frac{2}{\pi} \sin \left(\frac{\pi p}{2}\right) \int_{0}^{\infty} s^{-p} h(s) d s .
$$

Proof. By Tonelli's theorem,

$$
\int_{0}^{\infty} s^{-p} h(s) d s=\int_{0}^{\infty}\left(\int_{0}^{\infty} \frac{s^{1-p}}{s^{2}+u^{2}} d s\right) d \mu(u) .
$$

Letting $s=u t^{\frac{1}{2}}$, we find (using (5.8)) that

$$
\int_{0}^{\infty} \frac{s^{1-p}}{s^{2}+u^{2}} d s=\frac{1}{2} u^{-p} \int_{0}^{\infty} \frac{t^{-\frac{p}{2}}}{1+t} d t=\frac{\pi}{2}\left[\sin \left(\frac{\pi p}{2}\right)\right]^{-1} u^{-p} .
$$

This proves (5.10).

TheOREM 5.4. Let $(\mathcal{M}, \tau)$ and $z$ be as in Theorem 5.2, and let $n \in \mathrm{N}$.

(a) $z^{n}$ is an unbounded $R$-diagonal operator.

(b)

$$
\int_{0}^{\infty} \frac{s}{s^{2}+u^{2}} d \mu_{|z|^{n}}(u)=\left(s+s^{\frac{n-1}{n+1}}\right)^{-1}, \quad s>0 .
$$

(c) For $p \in\left(0, \frac{2}{n+1}\right), z^{n}$ and $z^{-n}$ both belong to $L^{p}(\mathcal{M}, \tau)$, and

$$
\left\|z^{n}\right\|_{p}^{p}=\left\|z^{-n}\right\|_{p}^{p}=\frac{(n+1) \sin \left(\frac{\pi p}{2}\right)}{\sin \left(\frac{(n+1) \pi p}{2}\right)} .
$$


(d) If $p \in\left(0, \frac{2}{n+1}\right)$ and $\lambda \in \mathrm{C}$, then $\operatorname{ker}\left(z^{n}-\lambda \mathbf{1}\right)=0$. Moreover, $\left(z^{n}-\right.$ $\lambda \mathbf{1})^{-1} \in L^{p}(\mathcal{M}, \tau)$ with

$$
\left\|\left(z^{n}-\lambda \mathbf{1}\right)^{-1}\right\|_{p} \leq\left\|z^{-n}\right\|_{p} .
$$

Proof. According to Proposition 3.9, $z^{n}$ is $R$-diagonal. Moreover, since

$$
\begin{aligned}
& \mathscr{S}_{\mu_{|z|^{2}}}(t)^{n}=\left(-\frac{t}{1+t}\right)^{n}, \quad t \in(-1,0), \\
& \chi_{\mu_{\left.k^{n}\right|^{2}}}(t)=\frac{1}{1+t} \mathscr{S}_{\mu_{\left.k^{n}\right|^{2}}}(t)=-\left(-\frac{t}{1+t}\right)^{n+1}, \quad t \in(-1,0),
\end{aligned}
$$

with inverse function

$$
\psi_{\mu_{\left.z^{n}\right|^{2}}}(u)=-\frac{(-u)^{\frac{1}{n+1}}}{1+(-u)^{\frac{1}{n+1}}}, \quad u \in(-\infty, 0) .
$$

Hence, for $\lambda \in(-\infty, 0)$,

$$
G_{\mu_{\left|z^{n}\right|^{2}}}(\lambda)=\frac{1}{\lambda}\left(1+\psi_{\mu_{\left.z^{n}\right|^{2}}}\left(\frac{1}{\lambda}\right)\right)=\frac{1}{\lambda\left(1+(-\lambda)^{-\frac{1}{n+1}}\right)} .
$$

Let

$$
h_{n}(s)=\int_{0}^{\infty} \frac{s}{s^{2}+u^{2}} d \mu_{\left|z^{n}\right|}(u), \quad s \in(0, \infty) .
$$

Then

$$
h_{n}(s)=s \tau\left(\left(s^{2} \mathbf{1}+\left|z^{n}\right|^{2}\right)^{-1}\right)=-s G_{\mu_{\left|z^{n}\right|^{2}}}\left(-s^{2}\right) \stackrel{(5.14)}{=}\left(s+s^{\frac{n-1}{n+1}}\right)^{-1} .
$$

This proves (b).

Since $z=x y^{-1}$, where $(x, y)$ is a circular family, it is clear that $z^{-n} \sim z^{n}$ for all $n \in \mathrm{N}$. Hence, $\left\|z^{n}\right\|_{p}=\left\|z^{-n}\right\|_{p}$ for all $p>0$. Note that for $p>0$,

$$
\left\|z^{-n}\right\|_{p}^{p}=\tau\left(\left|z^{-n}\right|^{p}\right)=\tau\left(\left|\left(z^{n}\right)^{*}\right|^{-p}\right)=\tau\left(\left|z^{n}\right|^{-p}\right) .
$$

Thus, by Lemma 5.3, for $p \in(0,2)$,

$$
\left\|z^{-n}\right\|_{p}^{p}=\int_{0}^{\infty} u^{-p} d \mu_{\left|z^{n}\right|}(u)=\frac{2}{\pi} \sin \left(\frac{\pi p}{2}\right) \int_{0}^{\infty} s^{-p} h_{n}(s) d s .
$$

By application of (5.11) we find that

$$
\int_{0}^{\infty} s^{-p} h_{n}(s) d s=\int_{0}^{\infty} \frac{s^{-p-\frac{n-1}{n+1}}}{s^{\frac{2}{n+1}}+1} d s=\frac{n+1}{2} \int_{0}^{\infty} \frac{t^{-\frac{(n+1) p}{2}}}{1+t} d t .
$$


Then by (5.15) and (5.8), for $0<p<\frac{2}{n+1}$,

$$
\begin{aligned}
\left\|z^{-n}\right\|_{p}^{p} & =(n+1) \sin \left(\frac{\pi p}{2}\right)\left[\sin \left(\pi\left(1-\frac{(n+1) p}{2}\right)\right)\right]^{-1} \\
& =(n+1) \sin \left(\frac{\pi p}{2}\right)\left[\sin \left(\frac{(n+1) \pi p}{2}\right)\right]^{-1},
\end{aligned}
$$

and this proves (c). Note that the right-hand side of (5.16) converges to $\infty$ as $p \rightarrow \frac{2}{n+1}-$. Hence, $z^{-n} \notin L^{\frac{2}{n+1}}(\mathcal{M}, \tau)$, and the same holds for $z^{n}$. In particular, $z^{n}$ is not bounded, and this proves (a). In order to prove (d), let $\lambda \in \mathrm{C} \backslash\{0\}$, and put

$$
h_{n, \lambda}(t)=\int_{0}^{\infty} \frac{t}{t^{2}+u^{2}} d \mu_{\left|z^{n}-\lambda \mathbf{1}\right|}(u), \quad t>0 .
$$

Then by Proposition 4.13,

$$
h_{n, \lambda}(t)=h_{n}\left(s_{n}(|\lambda|, t)\right), \quad t>0,
$$

where $s_{n}(|\lambda|, t)$ is given by Definition 4.9 in the case $\mu=\mu_{\left|z^{n}\right|}$. Note that, according to Definition 4.9,

$$
s_{n}(|\lambda|, t)>t, \quad t>0 .
$$

Moreover, by (5.11), $h_{n}$ is monotonically decreasing on $(0, \infty)$. Thus,

$$
h_{n, \lambda}(t) \leq h_{n}(t), \quad t>0 .
$$

It now follows from Lemma 5.3 that for $p \in(0,2)$,

$$
\int_{0}^{\infty} u^{-p} d \mu_{\left|z^{n}-\lambda \mathbf{1}\right|}(u) \leq \int_{0}^{\infty} u^{-p} d \mu_{\left|z^{n}\right|}(u) .
$$

According to (c), the right-hand side of (5.17) is finite for $p \in\left(0, \frac{2}{n+1}\right)$. Hence, for such $p, \operatorname{ker}\left(z^{n}-\lambda \mathbf{1}\right)=0,\left(z^{n}-\lambda \mathbf{1}\right)^{-1} \in L^{p}(\mathscr{M}, \tau)$, and

$$
\left\|\left(z^{n}-\lambda \mathbf{1}\right)^{-1}\right\|_{p}^{p} \leq\left\|z^{-n}\right\|_{p}^{p} .
$$

Remark 5.5. Note that Theorem 5.4 (a) and (c) generalize Theorem 5.2 (a) and (c) to all $n \in \mathrm{N}$. It is not hard to generalize Theorem 5.2 (b) and (d) as well. One finds that the distribution of $\left|z^{n}\right|$ is given by

$$
d \mu_{\left|z^{n}\right|}(t)=\frac{2}{\pi} \frac{\sin \left(\frac{\pi}{n+1}\right)}{t\left(t^{\frac{2}{n+1}}+2 \cos \left(\frac{\pi}{n+1}\right)+t^{-\frac{2}{n+1}}\right) r} 1_{(0, \infty)(t)} d t,
$$


and the Brown measure of $z^{n}$ is given by

$$
d \mu_{z^{n}}(s)=\frac{1}{n \pi} \frac{|s|^{\frac{2}{n}-2}}{\left(1+|s|^{\frac{2}{n}}\right)^{2}} d \operatorname{Re} s d \operatorname{Im} s .
$$

We leave the details of proof to the reader.

\section{REFERENCES}

1. Aagaard, L., and Haagerup, U., Moment formulas for the quasinilpotent DT-operator, Internat. J. Math. 15 (2004), 581-628.

2. Biane, P., and Lehner, F., Computation of some examples of Brown's spectral measure in free probability, Colloq. Math. 90 (2001), no. 2, 181-211.

3. Brown, L. G., Lidskii's Theorem in the Type II Case, in Geometric Methods in Operator Algebras (Kyoto 1983), H. Araki and E. Effros (Eds.), Pitman Res. Notes Math. Ser. 123 (1986), 1-35.

4. Bercovici, H., Voiculescu, D., Free convolution of measures with unbounded support, Indiana Univ. Math. J. 42, No. 3 (1993), 733-773.

5. Dykema, K., and Haagerup, U., DT-Operators and decomposability of Voiculescu's circular operator, Amer. J. Math. 126 (2004), 121-189.

6. Fack, T., Kosaki, H., Generalized s-numbers of $\tau$-measurable operators, Pacific J. Math. 123 (1996), 269-300.

7. Fuglede, B., Kadison, R. V., Determinant theory in finite factors, Ann. of Math. 55 (1952), 520-530.

8. Folland, G. B., Real analysis, Modern Techniques and their Applications, John Wiley and Sons, 1984.

9. Haagerup, U., Larsen, F., Brown's spectral distribution measure for R-diagonal elements in finite von Neumann algebras, J. Funct. Anal. 176 (2000), 331-367.

10. Haagerup, U., and Schultz, H., Invariant subspaces of operators in a general $I I_{1}$-factor, Preprint (2006), http://front.math.ucdavis.edu/math.OA/0611256.

11. Haagerup, U., Thorbjørnsen, S., A new application of random matrices: $\operatorname{Ext}\left(C_{\mathrm{red}}^{*}\left(\mathrm{~F}_{2}\right)\right)$ is not a group, Ann. of Math. 162 (2005), 711-775.

12. Handbook of Tables for Mathematics, 4th ed., CRC-press (1975).

13. Hayman, W. K., Kennedy, P. B., Subharmonic Functions, Vol. I, Academic Press, London, 1976.

14. Larsen, F., Brown Measures and R-diagonal Elements in Finite von Neumann Algebras, Ph.D. Thesis, University of Southern Denmark, 1999.

15. Larsen, F., Powers of R-diagonal Elements, J. Operator Theory 47 (2002), 197-212.

16. Nica, A., Speicher, R., R-diagonal pairs - a common approach to Haar unitaries and circular elements, Fields Inst. Commun. (1997), 149-188.

17. Voiculescu, D., Circular and semicircular systems and free product factors, in Operator Algebras, Unitary Representations, Algebras, and Invariant Theory, Progr. Math. 92 (1990), 45-60. 
BROWN MEASURES OF UNBOUNDED OPERATORS AFFILIATED WITH . . 263

18. Voiculescu, D., Dykema, K., and Nica, A., Free Random Variables, CMR Monogr. Ser. 1 (1992).

DEPARTMENT OF MATHEMATICS AND COMPUTER SCIENCE

UNIVERSITY OF SOUTHERN DENMARK

CAMPUSVEJ 55

5230 ODENSE M

DENMARK

E-mail: haagerup@imada.sdu.dk,schultz@imada.sdu.dk 
